\title{
DOE/ER/40774_-TI
}

\section{University of Colorado}

Nuclear Theory

\section{Progress Report}

Distriaution of THIS DOCUMENT is UNLMMTED ph MASTER 


\section{DISCLAIMER}

This report was prepared as an account of work sponsored by an agency of the United States Government. Neither the United States Government nor any agency thereof, nor any of their employees, makes any warranty, express or implied, or assumes any legal liability or responsibility for the accuracy, completeness, or usefulness of any information, apparatus, product, or process disclosed, or represents that its use would not infringe privately owned rights. Reference herein to any specific commercial product, process, or service by trade name, trademark, manufacturer, or otherwise does not necessarily constitute or imply its endorsement, recommendation, or favoring by the United States Government or any agency thereof. The views and opinions of authors expressed herein do not necessarily state or reflect those of the United States Government or any agency thereof. 


\section{DISCLAIMER}

Portions of this document may be illegible electronic image products. Images are produced from the best available original document. 


\section{CONTENTS}

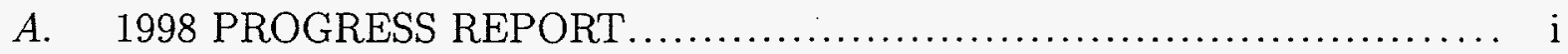

1. Nonresonant Contributions to Inelastic $N \rightarrow \Delta(1232)$ Parity Violation

2. Neutron Distribution Effects in Elastic Nuclear Parity Violation.......... 5

3. Wilson RG for scalar-plus-fermion field theories at finite density ........ 9

4. Perturbation theory for spin ladders using angular momentum coupled bases

5. Mean-field theory for spin ladders using angular momentum density ..... 16

6. Finite Temperature Renormalization Group Effective Potentials for the Linear Sigma Model.

7. Negative-Parity Baryon Resonances from Lattice QCD

8. The $N \rightarrow \Delta$ Electromagnetic Transition Amplitudes from QCD Sum Rules

9. Higher Nucleon Resonances in Exclusive Reactions $(\gamma, \pi N)$ on Nuclei 29

B. PUBLICATIONS AND REPORTS .................................... 32

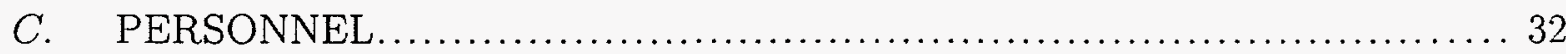


A.1 Non-resonant Contributions to Inelastic $N \rightarrow \Delta(1232)$ Parity Violation, S.J. Pollock, M.J. Ramsey-Musolf, H.W. Hammer.

If one measures a parity-violating (PV) asymmetry in electroproduction of the $\Delta$ using longitudinally polarized electrons on unpolarized nucleons, the resonant multipoles cancel, yielding a clean and simple structure. The $N \rightarrow \Delta$ transition is appealing for a PV experiment, since the $\Delta$ resonance is isolated from other nucleon resonances, the cross section is relatively large, and the transition is isovector. This isovector nature eliminates uncertainties associated with the unknown strangeness content of the nucleon, and yields a unique sensitivity to possible contributions from additional heavy particles not appearing in the Standard Model (SM). $N \rightarrow \Delta$ offers the additional advantage that it only couples strongly to one channel, $N \pi$, allowing one to treat unitarity issues quite rigorously. Our earlier work in this area focussed on experimental requirements to extract the axial response, and also quantified the limits on new physics (beyond the Standard Model) which could be obtained from this observable. Here, we discuss some fundamental theoretical limitations for extracting the above two pieces of physics [1].

We write the asymmetry as

$$
A_{L R}=\frac{N_{+}-N_{-}}{N_{+}+N_{-}}=\frac{-G_{\mu}}{\sqrt{2}} \frac{\left|q^{2}\right|}{4 \pi \alpha}\left[\Delta_{(1)}^{\pi}+\Delta_{(2)}^{\pi}+\Delta_{(3)}^{\pi}\right],
$$

$\Delta_{(1)}^{\pi}$ and $\Delta_{(3)}^{\pi}$ contain the Standard Model and axial physics of interest, respectively. The primary theoretical uncertainties arise from $\Delta_{(2)}^{\pi}$, which contains residual contributions from non-resonant vector-current isoscalar backgrounds. In order to quantitatively estimate the scale of these background contributions, we have used a model [4] to estimate non-resonant single pion production contributions to $A_{L R}$. Some detailed numbers can be found in table I. E.g., at $\theta=10^{\circ}$ and $\epsilon=1 \mathrm{GeV}$, we find $\Delta_{(2)}^{\pi} / \Delta_{(3)}^{\pi} \approx 6 \%$, so even a large uncertainty in $\Delta_{(2)}^{\pi}$ has negligible effect on an extraction of the axial term. Figure 1 shows the size of the backgrounds relative to the other contributions for forward angle scattering, as a function of momentum transfer. At kinematics suited for a determination of $\sin ^{2} \theta_{W}$, the vector-current background contributes $4-6 \%$ of the total asymmetry. A probe for new physics at the $1 \%$ level would require a theoretical uncertainty in $\Delta_{(2)}^{\pi}$ to be below $15-25 \%$, which may be difficult to achieve.

In the limit of single vector boson exchange between the electron and target, vector current conservation protects $\Delta_{(1)}^{\pi}$ from receiving large hadronic effects. The reason is that for this term, $J_{\mu}^{N C}$ and $J_{\mu}^{E M}$ differ only by a constant of proportionality $\left(\xi_{V}^{T=1}\right)$. Consequently, in the hadronic ratio $W^{P V} / W^{E M}$ most hadronic contributions to electroweak corrections cancel. This cancellation is not exact, due to light quark loop effects in the $Z^{0}-\gamma$ mixing tensor. For the present purposes, however, the level of uncertainty associated with these light quark loops appears to be negligible [6].

Additional corrections which remain to be estimated involve radiative corrections including two-boson exchange "dispersion corrections" (Fig 2a) and corrections induced by PV quark-quark interactions in the hadronic vertex. (Fig 2b) The former enter the analysis of all of the $\Delta_{(i)}^{\pi}$. These radiative corrections depend on the sum of products of transition current matrix elements, and the simple cancellation in $W^{P V} / W^{E M}$ that occurs in the oneboson exchange limit no longer applies [5]. The scale of this correction, sometimes referred 
to in the literature as a "dispersion correction", is nominally $\mathcal{O}(\alpha)$. Consequently, in order to extract constraints on new physics given $\xi_{V}^{T=1}$ to one percent, one must have a reliable calculation of the dispersion correction. To date, no such calculation has been carried out. An estimate for elastic PV processes has been reported by the authors of Ref. [6], where only the contribution from the nucleon intermediate state has been included. The results indicate that this contribution alone is not likely to be problematic. A complete calculation, for both elastic and inelastic processes, awaits future efforts.

The second radiative correction mentioned above (see Fig $2 \mathrm{~b}$ ) contributes to $\Delta_{(3)}^{\pi}$ only, at leading order. These effects involve parity-violating quark-quark interactions within the hadron, leading to an effective PV photon-hadron coupling [7]. One might call such effects a "transition anapole moment", in analogy with the static (elastic) anapole moment of the nucleon. Naïvely, these corrections are $\mathcal{O}(\alpha)$, and should not seriously affect a $25 \%$ determination of the axial vector transition form factors. This naïve scale, however, is somewhat misleading. The full PV amplitude containing these corrections is no longer proportional to $g_{V}^{e}$, as in the tree-level $Z^{0}$-exchange case, since a photon is now exchanged. Hence, relative to the tree-level process, the amplitude involving hadronic PV is of order $\alpha /\left|g_{V}^{e}\right| \approx 10 \alpha$. Furthermore, in the case of elastic PV electron scattering from the nucleon, the PV hadronic vertex receives additional infrared enhancements [7]. Should these enhancements appear in the $N \rightarrow \Delta$ case as well, the scale of the correction could be as large as the tree-level process. An estimate of such hadronic PV corrections has not yet been completed, but will be essential for extracting axial matrix element information from $\Delta_{(3)}^{\pi}$.

\begin{tabular}{cccccccc}
\hline \hline$E(\mathrm{GeV})$ & $\theta_{\mathrm{lab}}\left({ }^{\circ}\right)$ & $Q^{2}\left(\mathrm{GeV}^{2}\right)$ & $10^{5} A_{\text {tot }}$ & $\frac{\delta A_{\text {stat }}(\%)}{A_{\text {tot }}(\%}$ & $\frac{\Delta_{(2)}^{\pi}}{\Delta^{\pi}}(\%)$ & $\frac{\Delta_{(3)}^{\pi}}{\Delta^{\pi}}(\%)$ & $\frac{A_{A_{3}}}{\delta A_{\text {stat }}}$ \\
\hline .5 & 10. & .002 & -.03 & 45.9 & -.24 & 20.4 & .4 \\
1.0 & 10. & .020 & -.22 & 5.9 & -.7 & 11.0 & 1.9 \\
4.0 & 10. & .418 & -4.17 & .7 & -5.5 & 2.6 & 4.0 \\
\hline .5 & 90. & .106 & -1.24 & 10.9 & -2.81 & 17.1 & 1.6 \\
1.0 & 90. & .641 & -6.79 & 5.2 & -6.4 & 8.3 & 1.6 \\
4.0 & 90. & 5.566 & -54.46 & 26.8 & -13.4 & .8 & .0 \\
\hline .5 & 180. & .157 & -1.81 & 11.6 & -3.70 & 15.5 & 1.3 \\
1.0 & 180. & .846 & -8.85 & 7.5 & -7.0 & 7.1 & .9 \\
4.0 & 180. & 6.150 & -60.08 & 43.8 & -10.4 & .6 & .0 \\
\hline \hline
\end{tabular}

TABLE I. Our estimates of $A_{L R}$, statistical uncertainty for $A_{L R}$ (given our experimental assumptions), vector-current backgrounds, contribution of axial multipoles, and ratio of axial contribution to statistical uncertainty, respectively. $\Delta^{\pi}=\Delta_{(1)}^{\pi}+\Delta_{(2)}^{\pi}+\Delta_{(3)}^{\pi} ; A_{(3)}$ is the contribution to the $A_{L R}$ arising from $\Delta_{(3)}^{\pi}$. 


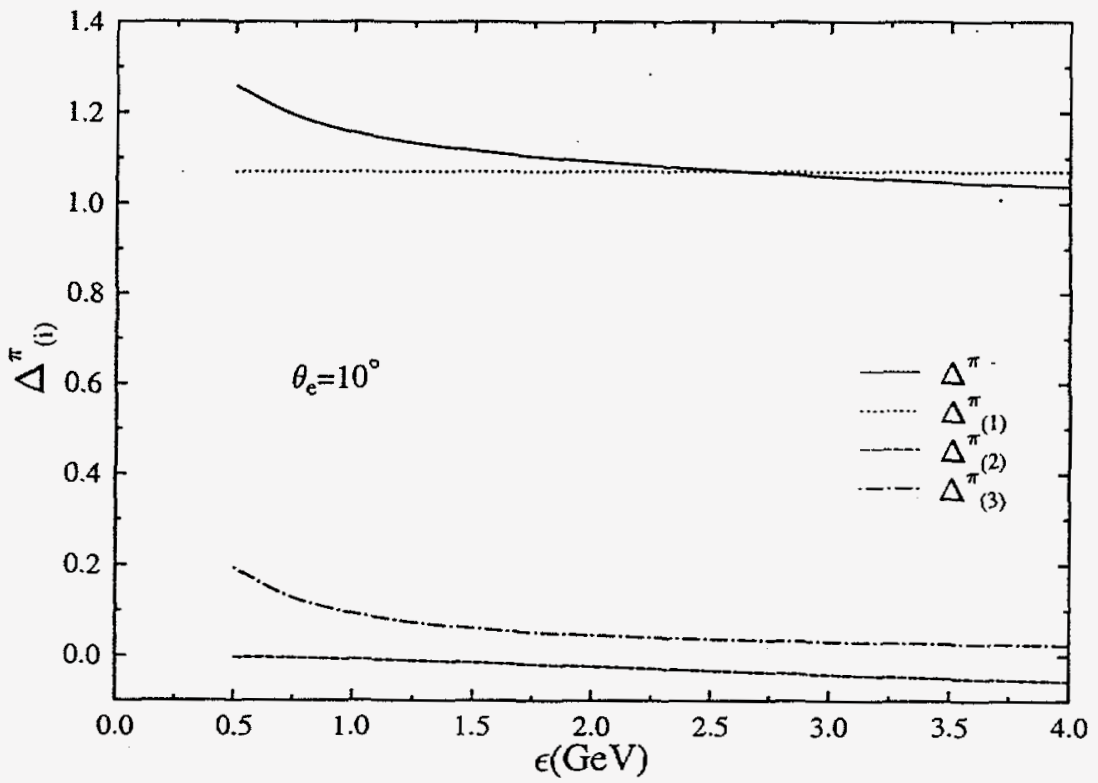

FIG. 1. Contributions to the asymmetry from the $\Delta_{(i)}^{\pi}$ defined in the text as a function of incident energy for $\theta=10^{\circ}$. Here, $\Delta^{\pi}=\Delta_{(1)}^{\pi}+\Delta_{(2)}^{\pi}+\Delta_{(3)}^{\pi}$.

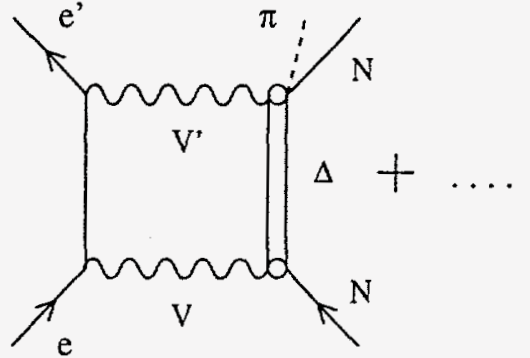

(a)

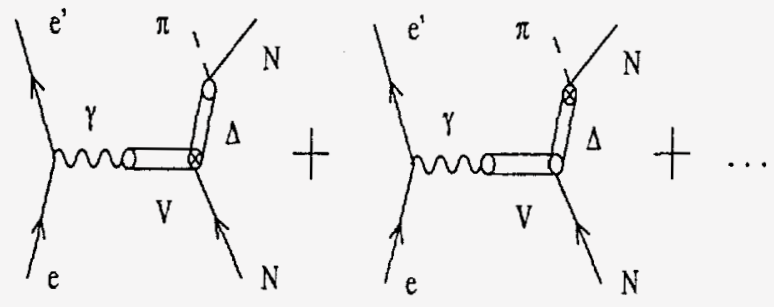

(b)

FIG. 2. Higher order hadron structure-dependent electroweak corrections to the $A_{L R}$. Fig (a) shows two-boson exchange "dispersion correction", where $V, V^{\prime}=\gamma, Z^{0}, W^{ \pm}$. Fig (b) illustrates corrections arising from hadronic parity violation. Open circles indicate a parity-conserving coupling, $\otimes$ denotes a weak, PV coupling. $V$ indicates a vector meson such as the $\rho$ or $\omega$. 
[1] N.C. Mukhopadhyay, M.J. Ramsey-Musolf, S. Pollock, J. Liu, and H.-W. Hammer. Nuclear Physics A633 (1998) 481. (nucl-th/9801025)

[2] M. J. Musolf et al., Phys. Rep. 239, 1 (1994).

[3] Jefferson Lab proposal E97-104, S.P. Wells, N. Simicevic, and K. Johnston.

[4] H. -W. Hammer and D. Drechsel, Z. Phys. A353, 321 (1995)

[5] M. J. Musolf and T. W. Donnelly, Z. Phys. C57, 559 (1993)

[6] W. J. Marciano and A. Sirlin, Phys. Rev. D29, 75 (1984)

[7] M. J. Musolf and B. R. Holstein, Phys. Rev. D43, 2956 (1991), Phys. Lett. B242, 461 (1990) 


\section{A.2 Neutron Distribution Effects in Elastic Nuclear Parity Violation, S.J. Pollock} and M. Welliver.

Parity violating electron scattering provides a unique method to determine spatial neutron distributions in heavy nuclei. Knowledge of the neutron distribution is of fundamental interest in nuclear structure models, and the first moment will be of interest in precision atomic parity experiments in the foreseeable future. The use of elastic scattering eliminates many complicated background issues, and the lack of magnetic and axial responses for $J=0$ targets simplifies the result [1]. Here, we have considered the parity violating asymmetry for barium and lead nuclei, which both have spin 0 and $N \neq Z$, and have investigated the relation between this observable, the moments of the neutron distibution, and the related atomic PNC observable.

It has been known for some time [2] that the simple result for $A\left(0^{+} 0 \rightarrow 0^{+} 0\right)$ involving a complete cancellation of nuclear structure is only valid for pure isoscalar transitions. In heavy nuclei, with $T \neq 0$, the asymmetry is modified, becoming

$$
A=A^{0}\left(\left(1-4 \sin ^{2} \theta_{W}\right)-\frac{\int j_{0}(q r) \rho_{n}(r) d^{3} r}{\int j_{0}(q r) \rho_{p}(r) d^{3} r}\right)
$$

where $\mathrm{q}$ is the momentum transfer, $j_{0}$ is a Bessel function, $\rho_{n(p)}$ is the spatial distribution of neutrons (protons), and $A^{0}=-G_{f}\left|q^{2}\right| /(4 \pi \alpha \sqrt{2})$. The denominator of the dominant final term in Eq. 1 is well known from elastic EM scattering. Thus, $A$ provides a clean direct measure of the neutron distribution.

In figure 1 we show the asymmetry as a function of $q^{2}$ for Barium, assuming a neutron distribution identical to protons (the smooth, solid curve) and also with $R_{n}=1.05 R_{p}$, for two different parameterizations of the shape (uniform distribution, and a two-parameter Fermi shape). These curves shows dramatic deviation from the solid (or naive Standard Model) curve, and show the clear sensitivity to isospin breaking, i.e. the neutron-proton distribution differences in this system. Figure $2 \mathrm{a}$ shows the predicted statistical error in a thousand hour experiment, with an arbitrary but plausible luminosity and solid angle. The statistical error decreases with the square root of the number of counts. The two curves have different assumed $R_{n}$; the statistical uncertainty is clearly not very sensitive to this quantity. In figure $2 \mathrm{~b}$, we show the uncertainty in the extracted neutron RMS radius (assuming the shape is identical to the proton shape aside from the radius) arising solely from the statistical uncertainties of figure $2 \mathrm{a}$. The two curves are for different assumed shapes, and again, the deviations due to shape are fairly small, particularly at lower q. For larger values of q, differences between neutron and proton shape become amplified by the Fourier transform and details of the distribution become more important in trying to fix the kinematics of the experiment.

We find, naturally, that very small momentum transfers $\left(q<0.2 \mathrm{fm}^{-1}\right)$ would provide a measurement least sensitive to the detailed shape of the neutron distribution. The predicted statistical errors for the asymmetry tend to be quite small at such values, due to the increasing elastic cross section at forward angles, but the value of the asymmetry is extremely small (typically $<5 \cdot 10^{-8}$ ), which means systematic errors will have to be extremely well controlled. Reaching such small values of $q$ also requires very forward angles $\left(<3^{\circ}\right)$, which is experimentally difficult. Although the asymmetry at low q depends on the neutron RMS 
radius (higher moments are suppressed by increasing powers of $q^{2}$ ) the dependence is itself explicitly suppressed by one power of $q^{2}$. We conclude that a single low momentum measurement will not effectively constrain moments of the neutron distribution, leaving considerable uncertainty in the resulting prediction for the nuclear radius correction to the atomic PNC observable.

At intermediate momenta $\left(0.2<q<0.6 \mathrm{fm}^{-1}\right)$, it is relatively easy to quantify and estimate the influence of the neutron distribution via a moment analysis. This allows for a more direct connection to atomic observables. The asymmetry itself is still quite small in this regime, and the figure of merit is worse than for the lower $q$ case, but the relative sensitivity to the first moment (RMS neutron radius) is optimized in this regime. At a value of about $q=0.52 \mathrm{fm}^{-1}$ for barium the uncertainty in rms neutron radius reaches a minimum value of about $3 \%$, given our experimental assumptions above. As seen in figure $2 b$, this minimum uncertainty and the q-value at which it is reached are largely independent of the shape of the neutron distribution.

At still higher momenta, $\left(0.6 \mathrm{fm}^{-1}<\mathrm{q}\right)$ the asymmetry grows, and the figure of merit tends upwards, with minima located between the elastic scattering diffraction minima. Here, however, the sensitivity to the shape is complicated and difficult to parameterize; a single measurement is not sufficient to constrain the atomic observables.

Once the uncertainty in the rms neutron radius is fixed from PV $\left(\vec{e}, e^{\prime} N\right)$ scattering, we can calculate a resulting uncertainty in the extraction of the weak mixing angle from atomic PV experiments. We find that a $3 \%$ measurement of the neutron radius could constrain $\sin ^{2} \theta_{w}$ at the $0.3 \%$ level if all other sources of uncertainty are small. Results for barium and lead are summarized in table. Our work in the near future will focus on extending these results to include the shape of the distributions, not just the RMS radius, further quantifying the relationship between the nuclear asymmetry ( $A_{L R}$ discussed here) and the related atomic PV observables in the same system [4].

Our results incorporate isospin breaking only in the different assumed neutron and proton spatial distributions. A charge symmetry breaking Hamiltonian which mixes isospin states at the nuclear level will further modify the predicted asymmetry. Some earlier estimates of these effects has been made in [2] for lighter nuclei. Naively, one expects these effects to be small, $\mathcal{O}(1 \%)$, but the calculations should be extended to the heavy nuclei we are considering. The additional effect of Coulomb distortion of the incoming electrons has been considered by Horowitz [3], and would modify our curves slightly.

\begin{tabular}{clllr}
\hline \hline Target & $\delta R_{n} / R_{n}$ & $\delta \sin ^{2} \theta_{W} / \sin ^{2} \theta_{W}$ & $q\left(\mathrm{fm}^{-1}\right)$ & $\delta A_{L R} / A_{L R}$ (stat) \\
\hline $\mathrm{Ba}$ & $3.1 \%$ & $0.3 \%$ & 0.52 & $11 \%$ \\
$\mathrm{~Pb}$ & $2.0 \%$ & $0.2 \%$ & 0.48 & $9 \%$ \\
\hline \hline
\end{tabular}

TABLE I. Target, minimum uncertainties in rms neutron radius and $\sin ^{2} \theta_{w}$, the associated momentum-transfer, and the statistical uncertainty in the asymmetry, given our experimental assumptions, and assuming uniform neutron and proton distributions. 


\section{Barium}

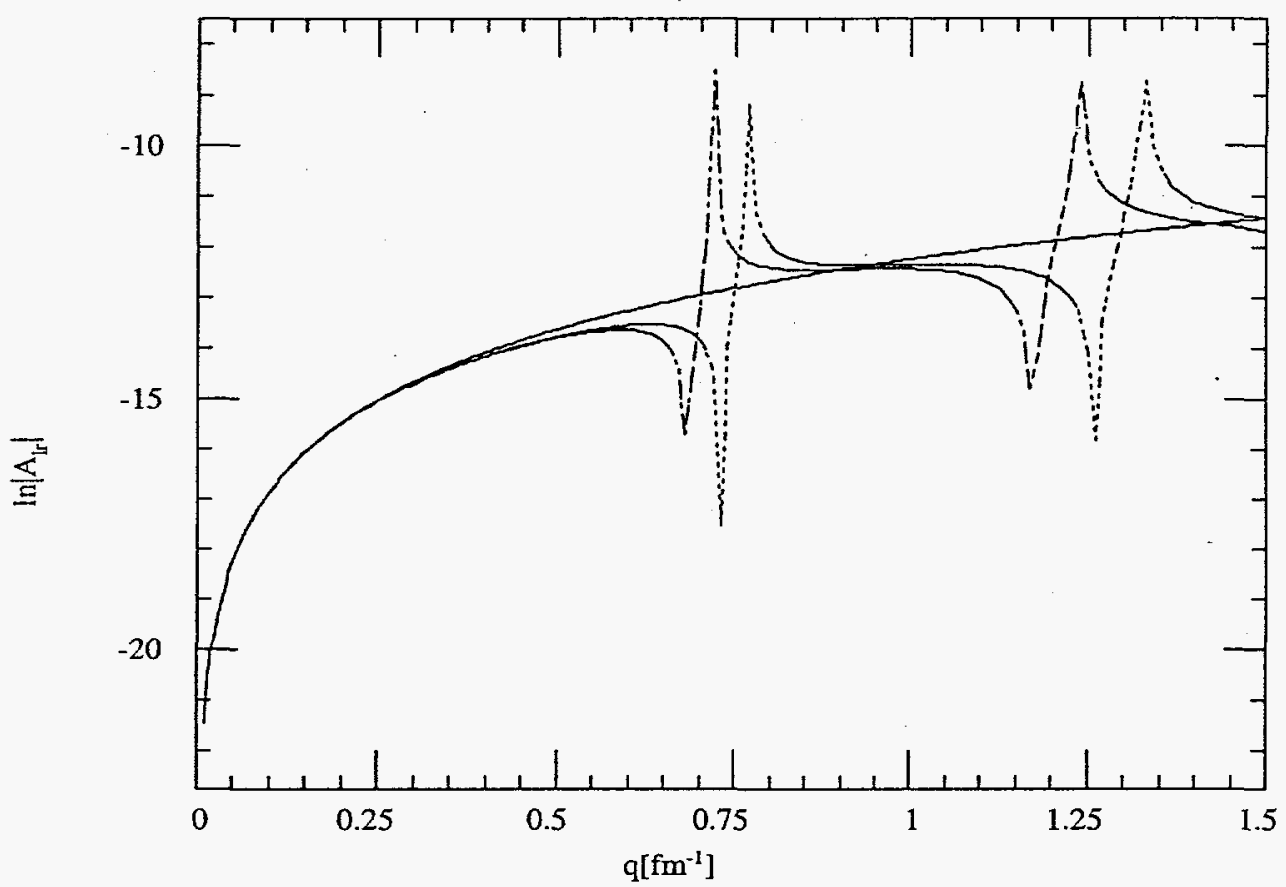

FIG. 1. Asymmetry versus momentum transfer, for Barium. Solid curve: identical neutron and proton distributions; long dashed curve: neutron radius $5 \%$ larger than proton radius; dashed curve: non-uniform (but identical) two-parameter Fermi shape ( $2 \mathrm{pF}$ ) for neutrons and protons.

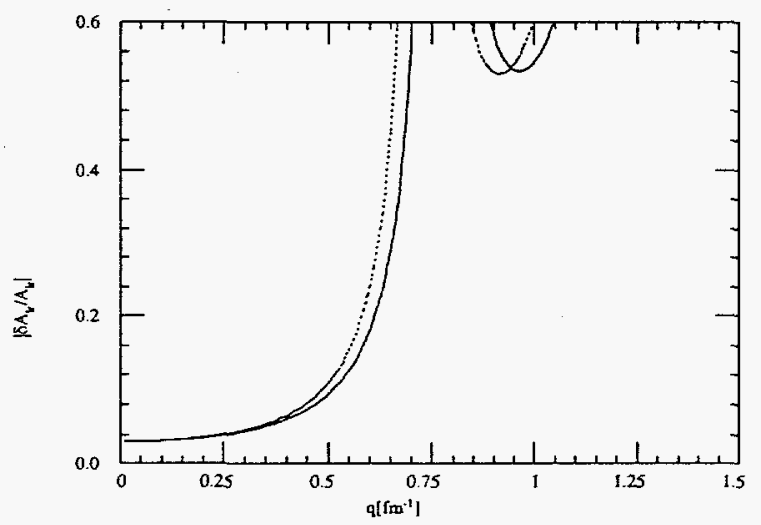

(a)

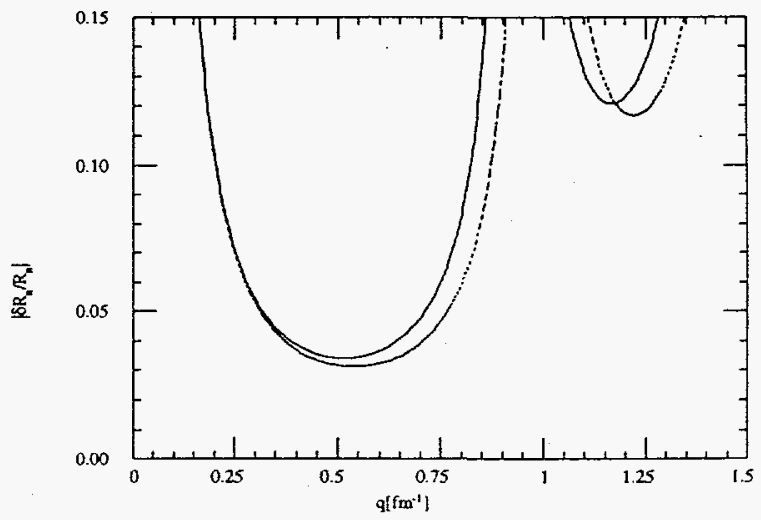

(b)

FIG. 2. a) Estimated statistical uncertainties in $A_{L R}$ on Barium, with a $100 \%$ polarized beam running 1000 hours with luminosity $2 \cdot 10^{36} / \mathrm{s} / \mathrm{cm}^{2}$, solid angle $10 \mathrm{msr}$. Solid curve assumes identical neutron and proton shapes and radii, parameterized by a three-parameter Gaussian shape (3pG). Dashed curve has $R_{n}=1.05 R_{p}$. b) Estimated uncertainty in the extracted RMS neutron radius, arising from the statistical uncertainties shown in figure a. The solid curve assumes a $3 \mathrm{pG}$ shape, dashed curve is for a $2 \mathrm{pF}$ shape. 
[1] M.J. Musolf et al, Physics Reports 239, (1994) 1.

[2] T.W. Donnelly, J. Dubach, I. Sick, Nucl. Phys A 503, 1989) 589.

[3] C. Horowitz, Phys.Rev. C57 (1998) 3430.

[4] S. J. Pollock, E.N. Fortson, L. Wilets. Phys. Rev. C46(1992) 2587. 
A.3 Wilson RG for scalar-plus-fermion field theories at finite density, J.R. Shepard and J.A. McNeil (Colorado School of Mines)

We have recently developed treatments of strongly interacting field theories based on the Wilson renormalization group (RG) [1-3]. Specifically, we have examined theories with self-interacting boson fields Yukawa coupled to fermion fields. These previous studies have looked at both zero temperature and finite temperature cases but have always been limited to zero fermion density. In the present work, we lift this restriction and consider the effects of non-zero fermion density. As discussed in the accompanying proposal, the ultimate goal of this project is , e.g., to extend our earlier investigations of the linear $\sigma$ model where chiral symmetry is broken by finite quark masses to to account for finite quark densities. At sufficiently high densities, spontaneous symmetry breaking will no longer occur and the original (approximate) $O(4)$ symmetry of the theory will be realized in the Wigner-Weyl rather than the Goldstone mode.

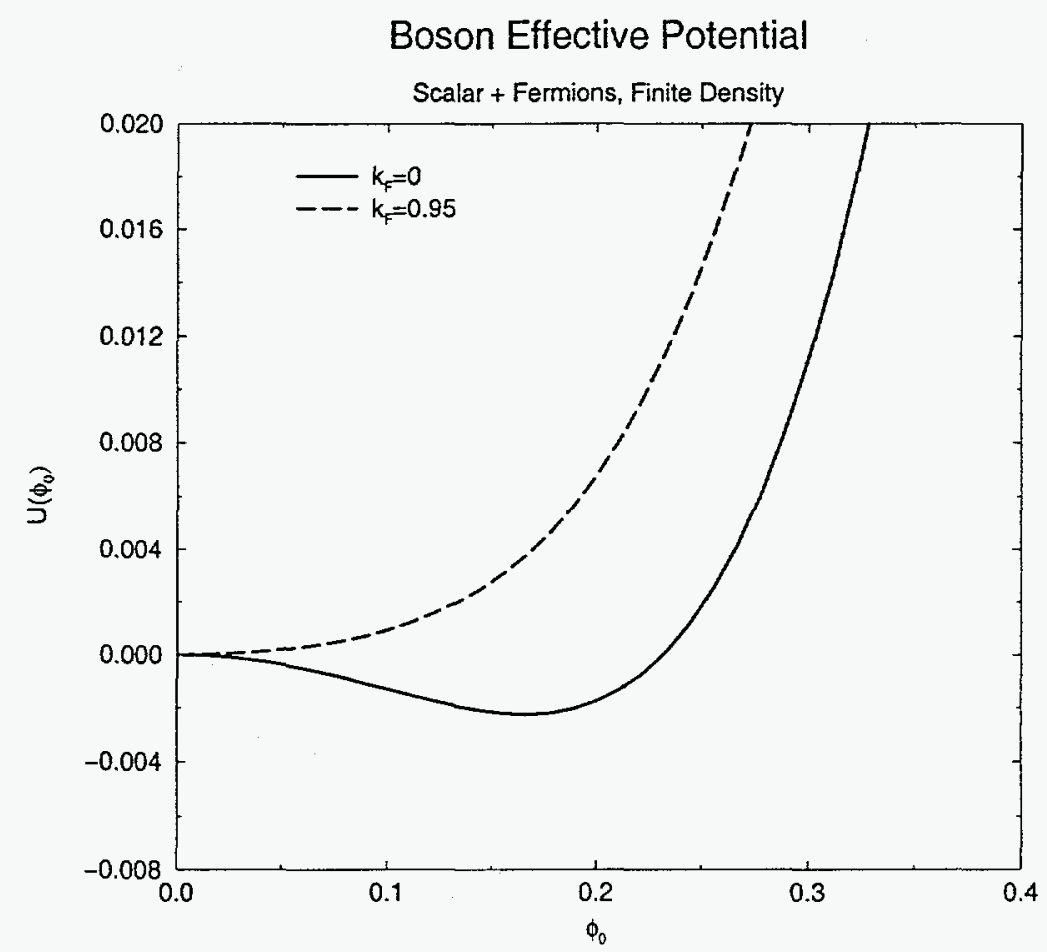

FIG. 1. Effective boson potential $U\left(\phi_{0}\right)$ for zero density $\left(k_{f}=0\right)$ and finite density $\left(k_{f}=0.95\right)$. Other parameters entering in these calculations are provided in the text. Note that finite density effects drive the solution toward the symmetric phase of the theory.

Here in our initial attempt to deal with finite density, we consider a single component self-interacting scalar field $\phi$ Yukawa coupled to a fermion field $\psi$ with $N_{f}$ flavors. We briefly sketch our main results. The original Euclidean action is

$$
S=\int d^{4} x\left\{\frac{1}{2}\left(\partial_{\mu} \phi\right)^{2}+U(\phi)+\bar{\psi}[i \gamma \cdot \partial-V(\phi)] \psi\right\} .
$$

In a more or less standard fashion $[4,5,3]$, we Fourier expand the boson and fermion fields about their uniform components, designated by $\phi_{0}$ and $z$, respectively. The exponentiated 
action is integrated over a thin shell of radius $\Lambda$ in the space of the non-uniform field components of $\phi$ and $\psi$. After projecting onto the uniform components only (i.e., making the Local Potential Approximation (LPA) [6]), we find the following RG flow equations for the boson potential $U\left(\phi_{0}\right)$ and the Yukawa coupling term $V\left(\phi_{0}\right)$ :

$$
\begin{aligned}
& \frac{d U}{d \Lambda}=-\frac{A_{d}}{2} \Lambda^{d-1}\left[\ln \left(\Lambda^{2}+U^{\prime \prime}\right)-N_{f} C_{d} \ln \left(\Lambda^{2}+V^{2}\right)\right. \\
& \left.+\frac{1}{\Lambda^{2}+V^{2}} \frac{4}{\pi}\left\{\frac{3 \Lambda^{2}}{4}\left[\theta_{\Lambda}-\frac{2}{3} \sin 2 \theta_{\Lambda}+\frac{1}{12} \sin 4 \theta_{\Lambda}\right]+m^{2}\left[\theta_{\Lambda}-\frac{1}{2} \sin 2 \theta_{\Lambda}\right]\right\}\right] \\
& \frac{d V}{d \Lambda}=-\frac{A_{d}}{2} \Lambda^{d-1} \frac{1}{\Lambda^{2}+U^{\prime \prime}}\left[V^{\prime \prime}-\frac{2 V\left(V^{\prime}\right)^{2}}{\Lambda^{2}+V^{2}}+\ldots\right]
\end{aligned}
$$

where $A_{d}=1 / 8 \pi^{2}$ and $C_{d}=4$ for Euclidean dimension $d=4$. The most important density dependent contribution corresponds to the last set of terms in the flow equation for $U$ which depends on $\theta_{\Lambda}$ defined via

$$
\theta_{\Lambda}= \begin{cases}\pi / 2, & \text { if } \Lambda<k_{f} \\ \sin ^{-1} k_{f} / \Lambda, & \text { if } \Lambda>k_{f}\end{cases}
$$

where $k_{f}$ is the Fermi momentum which specifies the fermion density. (The ellipses in the $V$ flow equation represent a complicated density dependent contribution which is numerically insignificant and will not be discussed further.) The form of this contribution can most readily be appreciated by considering the standard expression for the fermionic energy density associated with occupied positive energy states [7]:

$$
\mathcal{E}=\frac{1}{2} N_{f} C_{d} \int \frac{d^{3} p}{(2 \pi)^{3}} \sqrt{|\vec{p}|^{2}+m^{2}} \theta\left(k_{f}-|\vec{p}|\right)
$$

To reexpress $\mathcal{E}$ in $d=4$, we use the identity

$$
a=2 \int_{\infty}^{+\infty} \frac{d p_{0}}{2 \pi} \frac{a^{2}}{p_{0}^{2}+a^{2}}
$$

from which we readily obtain

$$
\mathcal{E}=N_{f} C_{d} \int \frac{d^{4} p}{(2 \pi)^{4}} \frac{p^{2} \sin ^{2} \theta_{2}+m^{2}}{p^{2}+m^{2}} \theta\left(k_{f}-p \sin \theta_{2}\right)
$$

where $\theta_{2}$ is the angle relative to the $p_{0}$ axis in $d=4$. Performing the angular integrations leads to

$$
\mathcal{E}=\frac{N_{f} C_{d}}{16 \pi^{2}} \int_{0}^{\infty} d p \frac{p^{3}}{p^{2}+m^{2}} \frac{4}{\pi}\left\{\frac{3 \Lambda^{2}}{4}\left[\theta_{p}-\frac{2}{3} \sin 2 \theta_{p}+\frac{1}{12} \sin 4 \theta_{p}\right]+m^{2}\left[\theta_{p}-\frac{1}{2} \sin 2 \theta_{p}\right]\right\}
$$

where

$$
\theta_{p}= \begin{cases}\pi / 2, & \text { if } p<k_{f} \\ \sin ^{-1} k_{f} / p, & \text { if } p>k_{f}\end{cases}
$$


Upon identifying $p \rightarrow \Lambda$ and $m \rightarrow V$, the integrand in Eqn. 7 corresponds to the density dependent contribution to the flow equation for $U$ appearing as Eqn. 2.

We solve the flow equations numerically as described in, e.g., Ref. [3]. Figure 1 shows the boson potential $U$ at zero density and at a density specified by $k_{f}=0.95$. Here the original UV cutoff is taken to be $\Lambda_{0}=\left(32 / \pi^{2}\right)^{1 / 4} \pi$ [1] and the IR cutoff is 0.571 . The UV boson potential is $U_{0}(\phi)=m_{0}^{2} \phi^{2} / 2+\lambda_{0} \phi^{4} / 4$ with $m_{0}^{2}=-2.57^{2}$ and $\lambda_{0}=10$. We have also taken $V_{0}(\phi)=3.5 \phi$ and $N_{f}=2$. Note that the value of $m_{0}$ has been tuned to yield a broken symmetry solution for zero density. The value of $k_{f}=0.95$ brings the system near the transition point between broken and symmetric phases. Such behavior can be understood qualitatively by recalling that the scalar field couples to the scalar density of the fermions. At zero density, only the (negative) scalar density associated with the sea enters. It yields an attractive interaction which opposes the repulsive self-coupling of the scalar field and drives the system toward the broken phase. The occupied positive energy states provide a positive contribution to the scalar density; roughly speaking, this contribution cancels the that from negative energy states of the same 3-momentum. Therefore the fermions have almost no effect on the flow for $\Lambda<k_{f}$ and the self-coupling of the scalar field again drives the system toward the symmetric phase. Note that the Yukawa potential $V$ flows very little at either zero or finite density.

We are in the process of extending this treatment to address the linear $\sigma$ model [3] where we will be able to estimate the physical density at which the transition to the symmetric phase of (nearly) massless quarks will occur. We also anticipate developing a finite temperature/finite density RG formulation.

[1] J.R. Shepard, V. Dmitrašinović and J.A. McNeil, Physical Review D51, 7017-7025 (1995); hep-lat/9412111.

[2] J.D. Shafer and J.R. Shepard, Physical Review D55, 4990-4996 (1997).

[3] A.S. Johnson, J.A. McNeil and J.R. Shepard, Physical Review D58:014001 (1998); hep-ph/9711370.

[4] M. Maggiore, Z. Phys. C41, 687 (1989)

[5] see T.E. Clark and S.T. Love, Phys. Lett. B344, 266 (1995) and references contained therein.

[6] see, e.g., T.E. Morris, hep-th/9802039.

[7] B.D. Serot and J.D. Walecka, Adv. in Nucl. Phys., Vol. 16, J. Negele and E. Vogt, eds., Plenum Press, N.Y., 1985. 
A.4 Perturbation theory for spin ladders using angular momentum coupled bases, J.R. Shepard and J. Piekarewicz (SCRI/FSU)

Spin ladders are currently the objects of intense scrutiny because of the possibility that they may afford insights into the dynamics of high temperature superconductors $[1,2]$. They have been studied using an astonishingly wide variety of theoretical techniques. Among these are a number of relatively simple, often analytic, treatments whose role is to help elucidate the physics underlying the numerical results of, e.g., direct diagonalization or density-matrix renormalization group (DMRG) calculations. These approaches are typically based on perturbation theory and almost invariably assume a "dimer" or "rung" basis (see Fig. 1). In this basis, the intra-chain couplings are treated perturbatively and the resulting expansions are routinely assumed to be meaningful, even in the region where the inter-chain and intra-chain couplings are comparable $[3,4,1]$.
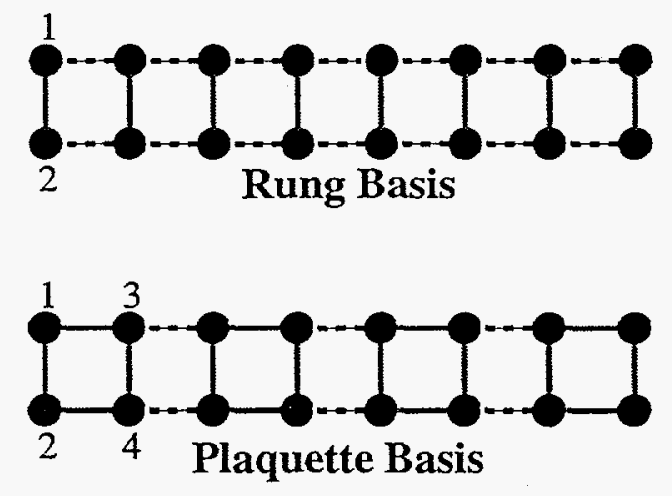

FIG. 1. Schematic representation of the rung and plaquette bases. The solid links represent those interactions which are diagonal in the basis; the dashed links represent the "perturbations".

We have developed a novel perturbative treatment of two-leg spin ladders implemented using a "plaquette" basis which, as we have argued previously [3,5], is particularly wellsuited to the study of spin ladders. Our method straightforwardly extracts perturbative information about the bulk system from ordinary Rayleigh-Schrödinger perturbation theory for finite systems. Here it is the "inter-plaquette" couplings which are treated perturbatively (see Fig. 1). In so doing we avoid the more general, and, thus necessarily more complicated, diagrammatic methods of "connected graph expansions" [8]. For example, we are able to conclude that the $n$-th order singlet ground-state energy of an $N$-plaquette ladder is generally expressible as:

$$
E_{J=0}^{(n)}(N)=A_{0}+B_{0} N
$$

where $A_{0}$ and $B_{0}$ are "intensive" coefficients, which are independent of $N$, for $N \geq n+1$. It is then clear that perturbative calculations for $n+1$-plaquette systems can yield the energy per site in the bulk limit, namely $B_{0} / 4$. Similar results allow us to determine bulk values for the spin gap and the one-magnon dispersion relations. Note that, at $n$-th order, the latter has the form

$$
\Delta^{(n)}(k)=\sum_{x=0}^{n} \Delta_{x}^{(n)} \cos (k x)
$$




\begin{tabular}{ccc}
\hline Order $n$ & $E_{0}^{(n)}$ & $\Delta^{(n)}$ \\
\hline 0 & -0.5 & 1 \\
1 & 0 & $-2 / 3$ \\
2 & -0.05815972 & 0.01967593 \\
3 & -0.01739728 & 0.06493538 \\
4 & -0.00329927 & 0.04306155 \\
5 & -0.00040692 & \\
6 & 0.00021569 & \\
7 & 0.00046854 & \\
8 & 0.00041483 & \\
9 & 0.00021860 & \\
10 & 0.00005215 & \\
11 & -0.00003610 & 0.46100619 \\
\hline Total & -0.57792948 & 0.504 \\
\hline DMRG & -0.57804314 & \\
\hline \hline
\end{tabular}

TABLE I. Plaquette-based perturbation theory for the bulk energy per site and spin gap of isotropic 2-leg spin ladders. DMRG results are from Ref. [2].

where $0 \leq k \leq 2 \pi$ is the linear momentum in lattice units. Expansion coefficients for the ground state energy per site appear in Table $I$.

Of course these methods can also be used with the rung basis. Calculations for a $2 \times 8$ ladder yield the following expansion for the ground state energy per site:

$$
E_{0} / N=-\frac{3}{8}-\frac{3}{16} \lambda^{2}-\frac{3}{32} \lambda^{3}+\frac{3}{256} \lambda^{4}+\frac{45}{512} \lambda^{5}+\frac{159}{2048} \lambda^{6}-\frac{55}{2048} \lambda^{7},
$$

while for the spin gap we obtain:

$$
\Delta=1-\lambda+\frac{1}{2} \lambda^{2}+\frac{1}{4} \lambda^{3}-\frac{1}{8} \lambda^{4}-\frac{35}{128} \lambda^{5}-\frac{157}{1024} \lambda^{6}+\frac{503}{2048} \lambda^{7} .
$$

The plaquette-basis perturbative results for the ground-state energy per site and for the spin gap appearing in Table I were obtained by carrying out Rayleigh-Schrödinger calculations for ladders as large as $2 \times 12$ (i.e., six plaquettes). These results allow us to determine the ground-state energy per site through 11 th order and the spin gap through fourth order. Through 11th order, the ground-state energy is within $0.02 \%$ of the DMRG [2] value while at fourth order, the perturbative estimate for the spin gap agrees with the DMRG value to better than $10 \%$.

A crucially important property of any perturbative series is its radius of convergence. In an effort to establish the radius of convergence quantitatively, we have compared high-order perturbative and exact results for finite ladders over a range of $\lambda$ values. For example, Fig. 2 shows the ground-state energy per site through 50th order for 4-, 5- and 6-plaquette systems as a function of $\lambda$. The radius of convergence is determined by the onset of wild excursions in these quantities at $\lambda_{c} \simeq 1.4$; the perturbative results are indistinguishable from the Lanczos 


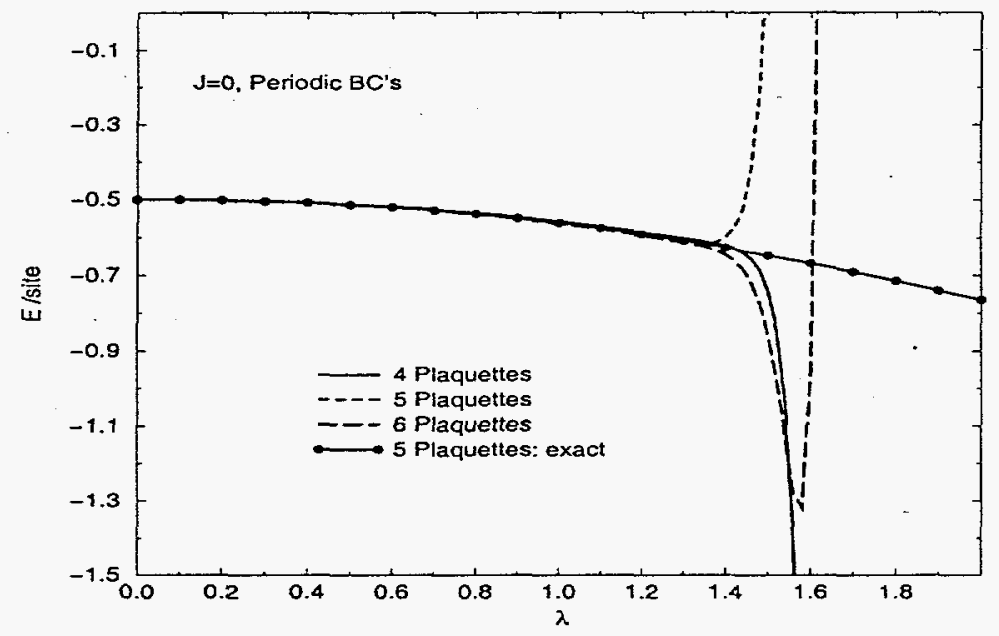

FIG. 2. Ground state energy per site for 4-, 5-, and 6-plaquette systems at 50th order in perturbation theory as a function of $\lambda$ which scales the inter-plaquette interaction (see the subsection on Rayleigh-Schrödinger Perturbation Theory). We conclude that the radius of convergence of the plaquette-basis perturbative expansion is about $\lambda_{c}=1.4$.

energies for $\lambda \leq \lambda_{c}$. Similar results are obtained for the lowest excited state, though the actual value of $\lambda_{c}$ is somewhat less, namely $\simeq 1.25$. As the value of $\lambda_{c}$ appears to have very little dependence on the size of the ladder, we assume it is also appropriate to the bulk system. It is gratifying that the physically interesting region of $\lambda=1$ lies well within the radius of convergence in this plaquette-basis treatment.

A similar analysis has been undertaken in the rung basis. The radius of convergence is again determined by the onset of wild excursions in the energy and is found to be $\lambda_{c} \simeq 0.8$ which does not include the physically interesting region of $\lambda=1$.

In conclusion, we have formulated perturbation theory for two-leg Heisenberg spin ladders in the "rung" and "plaquette" bases. We have been able to straightforwardly extract quantities appropriate to the bulk system from Rayleigh-Schrödinger perturbation theory for finite ladders. We find that the radius of convergence in the plaquette basis is $\lambda_{c} \simeq 1.25$ while, in the rung basis, it is $\lambda_{c} \simeq 0.8$. Clearly, the latter basis is strongly preferred when treating the physically interesting case of isotropic spin ladders which corresponds to $\lambda=1$ in both bases. As we have stressed elsewhere [3,5], the plaquette basis is especially wellsuited to the study of many aspects of spin ladders. Its success in the context of perturbation theory as presented here encourages us to use it in other investigations including a mean-field treatment of ladders (see, e.g., Ref. [1] for a rung-basis approach) and in a $t-J$ model aimed at the very interesting question of the dynamics of doped ladders (again, see, e.g., Ref. [10] for a rung-basis approach). Finally, we observe that use of the plaquette basis is enormously facilitated by relying on the powerful methods of Racah algebra which are well-known in nuclear physics but very rarely exploited in the condensed matter realm. Highlights of our examination of a plaquette-basis mean-field theory for spin ladders appear below in this Progress Report. Studies of the $t-J$ model are addressed in the accompanying proposal. 
[1] E. Dagotto and T.M. Rice, Science 271, 618 (1996).

[2] E. Dagotto, J. Riera and D.J. Scalapino, Phys. Rev. B 45, 5744 (1992).

[3] T. Barnes, E. Dagotto, J. Riera and E.S. Swanson, Phys. Rev. B47, 3196 (1993).

[4] M. Reigrotzki, H. Tsunetsugu and T.M. Rice, J. Phys. C 6, 9235 (1994); cond-mat/9408023.

[5] Sudha Gopalan, T.M. Rice and M. Sigrist, Phys. Rev: B49, 8901 (1994); cond-mat/9312026.

[6] J. Piekarewicz and J.R. Shepard, Phys.Rev. B56, 5366 (1997); cond-mat/9612139.

[7] J. Piekarewicz and J.R. Shepard, Phys.Rev. B57, 10260 (1998); cond-mat/9707207.

[8] Martin P. Gelfand, Rajiv R.P. Singh, and David A. Huse, Journal of Stat. Phys. 59, 1093 (1990) and references contained therein.

[9] S.R. White, R.M Noack and D.J. Scalapino, Phys. Rev. Lett. 73, 886 (1994).

[10] M. Sigrist, T.M. Rice and F.C. Zhang, Phys.Rev. B49, 12058 (1994); cond-mat/9401019. 
A.5 Mean-field theory for spin ladders using angular momentum coupled bases, J.R. Shepard and J. Piekarewicz (SCRI/FSU)

The previous section presented perturbation theory for spin ladders using both rung and plaquette bases. The rung-basis calculations were seen to have only limited value as the physically interesting region corresponding to $\lambda=1$ lies well outside the radius of convergence of the series for the ground state energy and the spin gap. Even for the plaquette basis calculations, where $\lambda=1$ is within the radius of convergence, considerable calculational effort is required to obtain reasonable accuracy for dynamical quantities. For these reasons, it is desirable to develop relatively simple non-perturbative methods for treating these strongly correlated systems. We have undertaken to fashion a mean-field theory for spin ladders. Our starting point is a familiar general expression for the second quantized Hamiltonian:

$$
\hat{H}=\sum_{\alpha \beta} c_{\alpha}^{\dagger}\langle\alpha|H| \beta\rangle c_{\beta} .
$$

In our notation, the rung basis Hamiltonian [1] is decomposed as $\hat{H}_{r u n g}=\hat{H}_{0}+\hat{V}$. Here $\hat{H}_{0}$ is the sum of all "intra-rung" interactions and can be written as

$$
\hat{H}_{0}=\sum_{i} \sum_{\substack{J_{i} M_{i} \\ J_{i}^{\prime} M_{i}^{\prime}}} A_{J_{i} M_{i}}^{\dagger}\left\langle J_{i} M_{i}\left|h_{0}(i)\right| J_{i}^{\prime} M_{i}^{\prime}\right\rangle A_{J_{i}^{\prime} M_{i}^{\prime}}
$$

where $A_{J_{i} M_{i}}^{\dagger} \equiv\left[c_{L}^{\dagger}(i) c_{R}^{\dagger}(i)\right]_{J_{i} M_{i}}$ and where $c_{i, L}^{\dagger}\left(c_{i, R}^{\dagger}\right)$ is the creation operator for the electron on the left (right) of the $i$-th rung and where $h_{0}(i)=\mathbf{s}_{L}(i) \cdot \mathbf{s}_{R}(i)$ with $\mathbf{s}_{L}(i)\left(\mathbf{s}_{R}(i)\right.$ ) being the spin operator for the electron on the left (right) of the $i$-th rung. Now $h_{0}$ is of course diagonal in the rung basis and the matrix elements of Eq. 2 are trivially evaluated. $\hat{V}$ is the sum of all "intra-rung" interactions and can be expressed as

$$
\hat{V}=\sum_{i} \sum_{\substack{J_{i} J_{i+1} \\ J_{i}^{\prime} J_{i+1}^{\prime}}} \sum_{J M}\left[A_{J_{i}^{\prime}}^{\dagger} A_{J_{i+1}^{\prime}}^{\dagger}\right]_{J M}\left\langle J_{i}^{\prime} J_{i+1}^{\prime} ; J M|V(i, i+1)| J_{i} J_{i+1} ; J M\right\rangle\left\{\left[A_{J_{i}}^{\dagger} A_{J_{i+1}}^{\dagger}\right]_{J M}\right\}^{\dagger} .
$$

where $V(i, i+1)=\mathbf{s}_{L}(i) \cdot \mathrm{s}_{L}(i+1)+\mathbf{s}_{R}(i) \cdot \mathbf{s}_{R}(i+1)$. The two-rung matrix elements in this expression are readily evaluated in terms of $9-j$ symbols.

If we now identify singlet $s_{i}^{\dagger} \equiv A_{J_{i}=M_{i}=0}^{\dagger}$ and triplet $t_{i, 1 M}^{\dagger} \equiv A_{J_{i}=1, M_{i}}^{\dagger}$ operators, then we can reexpress the two contributions to the Hamiltonian as $\hat{H}_{0} \rightarrow \sum_{i}\left[-3 / 4 \hat{s}_{i}^{\dagger} \hat{s}_{i}+1 / 4 \hat{\boldsymbol{t}}_{i}^{\dagger} \hat{\mathrm{t}}_{i}\right]$ and

$$
\hat{V} \rightarrow \sum_{i}\left\{\frac{1}{2}\left[s_{i}^{\dagger} s_{i+1}^{\dagger} \mathbf{t}_{i} \cdot \mathbf{t}_{i+1}+s_{i} s_{i+1} \mathbf{t}_{i}^{\dagger} \cdot \mathbf{t}_{i+1}^{\dagger}\right]+\frac{1}{2}\left[s_{i}^{\dagger} s_{i+1} \mathbf{t}_{i}^{\dagger} \cdot \mathbf{t}_{i+1}+s_{i+1}^{\dagger} s_{i} \mathbf{t}_{i+1}^{\dagger} \cdot \mathbf{t}_{i}\right]\right\}+\hat{V}_{4}
$$

where $\hat{V}_{4}$ contains contributions quartic in the $t_{i}$ 's. We now include an explicit constraint of the form $s_{i}^{\dagger} s_{i}+\mathrm{t}_{i}^{\dagger} \cdot \mathrm{t}_{i}=1$ to ensure that each rung is in either a singlet or a triplet state. The resulting expression for

$$
\hat{K} \equiv \hat{H}-\mu \sum_{i}\left(s_{i}^{\dagger} s_{i}+\mathrm{t}_{i}^{\dagger} \cdot \mathrm{t}_{i}-1\right),
$$

where $\mu$ is a Lagrange multiplier, is completely equivalent to that appearing as Eqns. 2.9-11 in Ref. [1] although the latter was obtained using very different methods. As will be shown 
below, our technique has the advantage that it may be readily extended to the plaquette basis.

At this point, we make the mean-field approximation which consists, namely $\left\langle s_{i}\right\rangle \rightarrow \bar{s}$. We also transform the triplet operators to momentum space using $\mathrm{t}_{i}^{\dagger}=N^{-1 / 2} \sum_{k} e^{i k r_{i}} \mathrm{t}_{k}^{\dagger}$ where $N$ is the total number of rungs and where we have implicitly assumed periodic boundary conditions. We furthermore assume that the triplet operators satisfy bosonic commutation relations. Dropping the quartic terms yields a quadratic form of $\hat{K}$ which can be diagonalized by a standard Holstein-Primakoff transformation: $\vec{\gamma}_{k}=\cosh \theta_{k} \mathbf{t}_{k}+\sinh \theta_{k} \mathrm{t}_{-k}^{\dagger}$. The transformed momentum-space form of $\hat{K}$ is

$$
\hat{K}=\left[(-3 / 4-\mu) \bar{s}^{2}+\mu\right] N+\frac{3}{2} \sum_{k}\left[\omega_{k}-\Lambda_{k}\right]+\sum_{k} \omega_{k} \vec{\gamma}_{k}^{\dagger} \cdot \vec{\gamma}_{k}
$$

where $\omega_{k}=\sqrt{\Lambda_{k}^{2}-4 \Delta_{k}^{2}}$ with $\Lambda_{k}=1 / 4-\mu+\vec{s}^{2} \cos k$ and $\Delta_{k}=\frac{\bar{s}^{2}}{2} \cos k$. It is important to note that this expression differs from the one appearing as Eqn. 2.16 in Ref. [1] in that the second term contains a factor of $3 / 2$ here rather than $1 / 2$ as is implicit in Ref. [1]. This factor appears as a consequence of the following identity for the triplet operators which follows directly from the assumed commutation relations: $\vec{\gamma}_{k} \cdot \vec{\gamma}_{k}^{\dagger}-\vec{\gamma}_{k}^{\dagger} \cdot \vec{\gamma}_{k}=3$. We reproduce the results of Ref. [1] exactly if the three on the rhs of this equation is replaced by one. As will become evident below, this difference has a profound effect on the final results. As usual, the ground state of the system is taken to be the vacuum for the $\vec{\gamma}_{k}$ operators. The ground state energy is simply the first two terms appearing in Eqn. 6 and the mean-field solution is specified by extremizing the energy with respect to $\vec{s}$ and $\mu$. Moreover, the onemagnon dispersion relation is specified by $\omega_{k}$ while the spin gap corresponds to $\omega_{k=\pi}$. We find $\mu=-1.4597$ and $\bar{s}^{2}=0.7813$; note that the latter quantity is the probability of finding any given rung in the interacting system in a singlet state. We then determine the energy per site of the ground state to be -0.5428 while the spin gap is 0.5015 . The corresponding values presented in Ref. [1] are -0.475 and $\simeq 0.1$, the differences arising from the factor of 3 in Eqn. 6 as discussed above. Our values compare remarkably well with the density matrix renormalization group (DMRG) [2] values of -0.57804314 and 0.504 .

We next reformulate the mean-field theory using the plaquette basis. As emphasized in Ref. [3] and in the preceding section of this report, the plaquette basis is well-suited to the study of isotropic (i.e., equal coupling along rungs and legs) spin ladders. In an important sense, the relative strength of "perturbations" - corresponding to inter-rung or interplaquette interactions in the two bases - are minimized in the plaquette basis. Consequently we expect the fully interacting ground state to more closely resemble the non-interacting state in the plaquette basis than in the rung basis and that mean-field theory should be a better approximation in the former.

The steps closely parallel the rung basis development given above and we only sketch them here. The plaquette analogue of $A$ as defined in the rung basis involves four $c$ 's and creates a plaquette with the angular momentum quantum numbers $L_{14}, L_{23}$ and $J M$ as discussed in Ref. [3]. Here the two L's are the "diagonal" spins which come from coupling opposite spins on a given plaquette and $J M$ are the total spin and spin-projection, respectively. Again the Hamiltonian is decomposed via $\hat{H}=\hat{H}_{0}+\hat{V}$ where $\hat{H}_{0}$ contains the sum of all "intra-plaquette" interactions while $\hat{V}$ accounts for the "inter-plaquette" interactions. The $\hat{H}_{0}$ is again quite simple; $\hat{V}$ is rather complicated but can be readily handled using the 
powerful methods of Racah algebra as presented in Ref. [3]. Indeed, the matrix elements which appear in the plaquette basis analogue of Eqn. 3 are precisely those appearing as Eqn. (7) of Ref. [3]. Moreover, as discussed at length there, the low energy states of the interacting spin ladders are dominated by the configurations in which the diagonal spins, i.e., $L_{14}(i)$ and $L_{23}(i)$, are triplets. We therefore truncate to such states. We now define new singlet and triplet operators which act in the truncated space (ignoring for now the quintet operators corresponding to $J=2$.) Now the singlet (triplet) operator annihilates a plaquette with $L_{14}=L_{23}=1$ and $J=0(J=1)$. We again make the mean-field approximation, transform to momentum space, drop terms quartic in the triplet operators and add the constraint requiring that each plaquette be either a singlet or triplet. The resulting quadratic expression for the plaquette analog of $\hat{K}$ can be diagonalized to yield the following expression:

$$
\hat{K}=\left[(-2-\mu) \vec{s}^{2}+\mu\right] N+\frac{3}{2} \sum_{k}\left[\omega_{k}-\Lambda_{k}\right]+\sum_{k} \omega_{k} \vec{\gamma}_{k}^{\dagger} \cdot \vec{\gamma}_{k}
$$

where now $\Lambda_{k}=-1-\mu-\frac{2 \bar{s}^{2}}{3} \cos k, \Delta_{k}=\frac{-2 \bar{s}^{2}}{3} \cos k, N$ is the total number of plaquettes, and $\omega_{k}=\sqrt{\Lambda_{k}^{2}-4 \Delta_{k}^{2}}$ as before. Once again, the mean-field solution for the ground state is obtained by extremizing the $c$-number terms of Eqn. 7 with respect to $\bar{s}$ and $\mu$. Note that $\vec{s}^{2}$ is now the probability of finding any given plaquette in the interacting system in a state where the diagonal spins are triplets and the overall spin is a singlet. Again, the one-magnon dispersion relation is given by $\omega_{k}$ but now the spin gap coincides with $\omega_{k=0}$. We find $\mu=-2.3568$ and $\bar{s}^{2}=0.8582$, the latter being considerably closer to one than in the rung basis, in keeping with our expectations. The energy per site and the spin gap are -0.5411 and 0.5369 , respectively. Perhaps surprisingly, both values agree slightly less well with DMRG than their rung-basis counterparts.

One plausible explanation for what appears to be a poorer than expected performance by the plaquette-basis calculation is that we have made a significant truncation which was not made in the rung-basis treatment. As discussed at length in Ref. [3], it is possible to systematically correct for truncation errors by constructing an effective low-energy Hamiltonian to be used with the restricted basis. We accomplish this via the recently developed COntractor REnormalization (CORE) method [4]. Here the effective Hamiltonian is expressed as a cluster expansion. It is straightforward to incorporate 2-plaquette CORE contributions in the present mean-field calculations. In effect, the matrix elements appearing in $\hat{V}$ are changed by factors ranging from a few to as much as $20 \%$. Also some new matrix elements appear; one consequence of these new matrix elements is that the ground state energy now contains a term with is quartic in the mean field, $\bar{s}$. We find that the CORE-mean-field ground state energy is extremized for values of $\mu=-2.6013$ and $\bar{s}^{2}=0.8500$. The energy itself is -0.5699 while the spin gap is 0.4770 . This represents a significant improvement in the energy over both the rung and non-CORE plaquette calculations.

We have attempted to investigate the accuracy of our mean-field formulations by evaluating the corrections to the energy and the spin gap arising from the terms in $\hat{K}$ which are quartic in the triplet operators (i.e., arise from $\hat{V}_{4}$ ) and which we have so far ignored. We add the perturbative contribution to the ground state energy, $\left\langle 0\left|\hat{V}_{4}\right| 0\right\rangle$, to the ground state energy and determine $\mu$ and $\bar{s}$ by extremizing the sum. We also evaluate the shift in the excitation energy of the one-magnon states due to $\hat{V}_{4}$. The results of these and all previously 


\begin{tabular}{|ccccc|}
\hline Calculation & $\mu$ & $\bar{s}^{2}$ & e/site & spin gap \\
\hline rung & -1.4597 & 0.7813 & $-0.5428(-6.10)$ & $0.5015(-0.50)$ \\
rung $+H_{2}$ & -1.5559 & 0.7465 & $-0.5776(-0.08)$ & $0.5511(+9.35)$ \\
\hline plaq & -2.3568 & 0.8582 & $-0.5411(-6.39)$ & $0.5369(+6.53)$ \\
plaq $+H_{2}$ & -2.3826 & 0.8429 & $-0.5447(-5.77)$ & $0.5519(+9.50)$ \\
\hline plaq + CORE & -2.6013 & 0.8500 & $-0.5699(-1.41)$ & $0.4770(-5.36)$ \\
plaq + CORE $+H_{2}$ & -2.6766 & 0.8123 & $-0.5801(+0.36)$ & $0.4372(-13.3)$ \\
\hline DMRG $[2]$ & & & -0.57804314 & 0.504 \\
\hline
\end{tabular}

TABLE I. Properties of mean-field solutions. In the last two columns, percentage departures in magnitude from the DMRG values appear in parentheses.

discussed calculations are summarized in Table I. We see that the changes in the energy per site and the spin gap due to $\hat{V}_{4}$ are much larger in the rung basis than in the plaquette basis. This is consistent with the general observation that inter-rung interactions are generally larger than inter-plaquette interactions or, equivalently, that the non-interacting plaquette ground state is a significantly better approximation to the true ground state than is the non-interacting rung ground state. It also suggests that the reasonably good agreement between the simplest rung-basis mean field theory and the DMRG is fortuitous and will not persist in better approximations. We also conclude that inclusion of CORE corrections is significant in the plaquette calculations. Agreement of the ground state energy with the DMRG is considerably improved by these contributions while values for the spin gap are changed substantially but not so as to move them closer to the DMRG. This indicates that our plaquette basis mean field theory could be substantially improved by making a less severe truncation. We are at present extending our approach to include plaquette triplets formed by coupling one diagonal spin to one and the other to zero, thus treating explicitly some of the dynamics included implicitly in CORE. We are also developing a self-consistent treatment of the $\hat{H}_{2}$ contributions. In any case, the mean field theories we have developed already provide a surprisingly good account of the properties of spin ladders considering their relative simplicity and minimal computational requirements.

[1] Sudha Gopalan, T.M. Rice and M. Sigrist, Phys. Rev. B49, 8901 (1994); cond-mat/9312026.

[2] S.R. White, R.M Noack and D.J. Scalapino, Phys. Rev. Lett. 73, 886 (1994).

[3] J. Piekarewicz and J.R. Shepard, Phys.Rev. B56, 5366 (1997); cond-mat/9612139.

[4] C.J. Morningstar and M. Weinstein, Phys. Rev. Lett. 73, 1873 (1994); Phys. Rev. D 54, 4131 (1996).

[5] J. Piekarewicz and J.R. Shepard, Phys.Rev. B57, 10260 (1998); cond-mat/9707207. 
A.6 Finite Temperature Renormalization Group Effective Potentials for the Linear Sigma Model. J.D. Shafer and J. R. Shepard (University of Colorado)

We have derived a solution for the linear sigma model at finite temperature, in the limit of the local potential approximation. This model was originally formulated by Schwinger [1] in 1958, but was subsequently popularized by Gell-Mann and Levy. Since that time, it has been thoroughly studied using a variety of techniques, and has recently enjoyed a resurgence of popularity as the "chiral quark meson" model $[2,3]$, in which the fermion fields correspond to valence quarks. The model has a rich spectrum of behavior, including spontaneous and dynamical symmetry breaking. However, for present purposes, we have considered only the case of finite temperature spontaneous chiral symmetry breaking, leaving the more complex case of finite temperature dynamical symmetry breaking for future work.

Since it is well known that the strong interactions in thermal equilibrium are significantly different at high temperature than at low temperature, the focus of our efforts has been the calculation of the critical temperature, $T_{c}$, at which chiral symmetry breaking spontaneously occurs. For example, a phase transition at some critical temperature may separate high and low temperature physics [4]. In particular, it was realized early on that such a transition should be closely connected to a qualitative change in the chiral condensate $\langle\bar{\psi} \psi\rangle$ in accordance with the observation that spontaneous symmetry breaking tends to be absent at sufficiently high temperatures. In addition, it has been pointed out that for small values of the up and down quark masses, the chiral transition is expected to share the universality class of the $O(4)$ Heisenberg model $[5,6]$.

Our solution consists of a system of coupled, nonlinear differential equations describing the change in the bosonic effective potential, $U$, and the Yukawa coupling, $g$, of the model as the energy scale of interest is lowered. These equations allow a numerical solution, and in this way we have been able to calculate renormalized couplings for the model at energy scales relevant to nuclear physics.

Our derivation begins with the finite temperature effective action given by

$$
\begin{aligned}
S_{E} & =\int_{0}^{\beta} d \tau \int d^{3} x\left\{\frac{1}{2}\left(\partial_{E} \phi\right)^{2}+\frac{1}{2}\left(\partial_{E} \vec{\pi}\right)^{2}+U(\rho)\right. \\
& \left.+\bar{\psi}\left[Z(\rho)\left(\gamma^{0} \frac{\partial}{\partial \tau}-i \vec{\gamma} \cdot \nabla\right)+V(\rho)\right] \psi\right\},
\end{aligned}
$$

where $\beta$ is the familiar reciprocal temperature from thermodynamics, and we have performed a Wick rotation to Euclidean space $[7,8]$. The fields are then expanded in terms of their Fourier components, which, together with a Taylor series expansion of the potentials, permits an evauation of the renormalization group flow relation for the model, given by

$$
e^{-S_{E}(\Lambda-\Delta \Lambda)}=\prod_{\vec{q}_{i}, n}^{\prime(\Lambda)} \int d \phi_{n}^{*}\left(\vec{q}_{i}\right) d \phi_{n}\left(\vec{q}_{i}\right) d \vec{\xi}_{n}^{*}\left(\vec{q}_{i}\right) d \vec{\xi}_{n}\left(\vec{q}_{i}\right) d \bar{\chi}_{n}\left(\vec{q}_{i}\right) d \chi_{n}\left(\vec{q}_{i}\right) e^{-S_{E}(\Lambda)}
$$

Then, in the limit of the local potential approximation, we finally acquire the following system of coupled differential equations:

$$
\frac{d U^{(\Lambda)}}{d \Lambda}=-\frac{A_{d-1}}{2 \beta} \sum_{n=-N}^{N} \Lambda\left(\Lambda^{2}-\omega^{2}\right)^{1 / 2}\left\{N_{f} C_{d} \ln \left[\Lambda^{2}+(4 n+1) \frac{\pi^{2}}{\beta^{2}}+g^{(\Lambda) 2} \rho^{2}\right]\right.
$$




$$
\begin{aligned}
&\left.-\ln \left(\Lambda^{2}+U^{\prime \prime(\Lambda)}\right)-(N-1) \ln \left(\Lambda^{2}+U^{\prime(\Lambda)} / \rho\right)\right\} \\
& \frac{d g^{(\Lambda)}}{d \Lambda}=-\frac{A_{d-1}}{2 \beta} \sum_{n=-N}^{N} \Lambda\left(\Lambda^{2}-\omega^{2}\right)^{1 / 2}\left\{\frac{1}{\Lambda^{2}+U^{\prime \prime(\Lambda)}}\right. \\
& \times\left(g^{\prime \prime}+2 \frac{g^{\prime}}{\rho}-2 \frac{\left(g g^{\prime 2} \rho^{2}+2 g^{2} g^{\prime} \rho+g^{3}\right)}{D_{F}}\right) \\
&\left.+\frac{(N-1)}{\Lambda^{2}+U^{(\Lambda) \prime} / \rho}\left[\frac{g^{\prime}}{\rho}+\frac{2 g^{3}}{D_{F}}\right]\right\}
\end{aligned}
$$

These equations exhibit the appropriate limiting behavior as $T \rightarrow \infty$, reducing to the correct expression for a theory with bosons only in $d-1$ dimensions. This is consistent with the phenomenon of dimensional reduction and the expected decoupling of fermions from our theory. Moreover, as $T \rightarrow 0$, we readily recover the zero temperature result of Ref. [9], derived using the local potential approximation for the linear sigma model, but at zero temperature.

In order to make to make physically meaningful predictions, it was necessary to constrain the free parameters of the model. It may be argued that one of the parameters most rigidly constrained by low energy data is the value of the pion decay constant, $f_{\pi}$. For this reason, we tuned the input bare scalar mass $\mu_{0}$ so that at $T=0$, the vacuum expectation value of the scalar field was $92.4 \mathrm{MeV}$, i.e., $\langle\phi\rangle=f_{\pi}$.

For our UV cutoff, we selected a value of $1 \mathrm{GeV}$ since at this energy the dynamics of the strong interactions begin to be dominated by nonlocal meson-like effective four quark interactions which are not computable by means of perturbative methods [10]. Moreover, below this scale, at approximately $600 \mathrm{MeV}$, it is thought that quarks are supplemented by additional degrees of freedom in the form of mesonic bound states. We note that this is well above the confinement scale of $\Lambda_{Q C D} \simeq 200 \mathrm{MeV}$, implying that at this scale there exists a mixture of quarks and mesons. Presumably, the meson dynamics may be described by light quarks with a Yukawa coupling, with leading order gluon effects accounted for by the formation of the mesonic bound states. It is expected that other hadronic bound states provide only subleading contributions to the dynamics since their masses are greater than those of the light scalar mesons. In addition, due to the decoupling of the quarks, and the entire colored sector of the theory with them, the matter of confinement likely has little influence on the meson dynamics at scales less than $300 \mathrm{MeV}$. We therefore set our IR scale at $135 \mathrm{MeV}$ since the flow of the couplings stops at scales below the pion mass.

This left us with two bare parameters unfixed: the quartic scalar coupling $\lambda_{0}$ and the Yukawa coupling $g_{0}$. Since preliminary work with scalar only models $[11,12]$ suggested that the values of $\lambda_{0}$ and $\mu_{0}$ are not entirely independent, we calculated $T_{c}$ for a variety of values of $\lambda_{0}$, tuning $\mu_{0}$ for each case to ensure that at $T=0,\langle\phi\rangle=f_{\pi}$. In this way we assessed the sensitivity of our value of $T_{c}$ with respect to $\lambda_{0}$.

Finally, although our zero-density model does not include nucleons, we know that the Goldberger-Treiman relation asserts that $g \simeq M_{N} / 3 f_{\pi} \simeq 3.7$. Thus, since we are aware that the quark-meson coupling is strong at $\Lambda_{I R}$, our calculations used values of $g_{0}$ such that renormalized values of $g$ were in the range $2.0<g<4.0$. We therefore assessed the variation of $T_{c}$ with $g_{0}$ for a physically relevant range of values of the renormalized coupling $g$. This is similar to the approach of Berges [10] which fixes $g_{0}$ and uses $M_{N}$ as phenomenological input. 
In this way, we have computed renormalized values of the scalar mass $m$, the quartic coupling $\lambda$, the Yukawa coupling $g$, and the expectation value of the scalar field $\langle\phi\rangle$, for temperatures ranging from 60 to $180 \mathrm{MeV}$. In addition, we observed the thermally driven phase transition which corresponds to the breaking of chiral symmetry. This allowed us to determine the critical temperature, $T_{c}$. The values we have obtained for this quantity range from about 115 to 135 $\mathrm{MeV}$, and compare favorably with the majority of theoretical and analytical work in this area $[10,13]$. Moreover, we have found that our values for $T_{c}$ are relatively robust in the face of significant variation of the phenomenologically determined input parameters of the model.

Plans for future work in this area are numerous since it is easy to see that our work could readily be extended in several ways. The most obvious extension is the consideration of dynamically broken chiral symmetry; i.e., the addition of explicit quark mass terms to the Lagrangian of Eq. 1. This would allow us to make calculations involving a nonzero pion mass, and would therefore permit the calculation of $\pi-\pi$ scattering lengths at finite temperature. In fact, this work has been done for the case of zero temperature by Johnson, Shepard and McNeil [9], and is of considerable algebraic complexity, even at $T=0$. It is therefore likely that consideration of this problem at $T \neq 0$ would constitute a major research effort, although it is almost certainly feasible.

Another possibility is the inclusion of finite density in our calculation, permitting an analysis of nuclear phenomena. We speculate that it might be possible to do this by phasing out the flow of fermionic modes in the neighborhood of the Fermi momentum, $k_{F}$, in accordance with the Fermi-Dirac distribution, with bosonic modes continuing to flow. In this way, we could presumably directly account for finite temperature effects.

It may also be possible to include strangeness in our work by extending it to $S U(3) \times S U(3)$. As remarked by Jungnickel and Wetterich [14], this would require the addition of two more field variants. Due to the significantly larger mass of the strange quark, this symmetry is much more broken in nature though it is still of real phenomenological utility.

Finally, also worth consideration is the application of our technique to NJL models at finite temperature [15], the well known Skyrme Lagrangian [16], or, in the interest of including specific mechanisms for the confinement characteristic of QCD, the MIT bag model [17]. Thus, there are a considerable number of phenomenological models, and individual variations of them, which may be investigated using our technique.

${ }^{1}$ J. Schwinger, Ann. Phys. (New York) 2, 407 (1958)

${ }^{2}$ M. Birse and M. K. Banerjee, Phys. Rev. D31, 118 (1985)

${ }^{3}$ S. Kahana, G. Ripka and V. Soni, Nucl. Phys. A415, 351 (1984)

${ }^{4}$ H. Meyer-Ortmanns, Rev. Mod. Phys. 68, 473 (1996)

${ }^{5}$ K. Rajgopal, in Quark-Gluon Plasma 2, edited by R. Hwa, World Scientific, Singapore (1995)

${ }^{6}$ C. Bernard, et al., Phys. Rev. D55, 6861 (1997)

${ }^{7}$ T. Matsubara, Prog. Theor. Phys. 14, 351 (1955).

${ }^{8}$ J.I. Kapusta, Finite-temperature field theory, Cambridge University Press, Cambridge 
(1989).

${ }^{9}$ A. S. Johnson, J. A. McNeil and J. R. Shepard, Report No. hep-ph-9711370 (submitted to Phys. Rev. D)

${ }^{10}$ J. Berges, D. U. Jungnickel and C. Wetterich, hep-ph/9705474 (1997)

11 J. R. Shepard, V. Dmitrašinović and J. A. McNeil, Phys. Rev. D51, 7017 (1995)

12 J.D. Shafer and J.R. Shepard, Phys. Rev. D55, 4990 (1997).

${ }^{13}$ F. Karsch, Report No. hep-lat-9709071 (unpublished)

${ }^{14}$ D. U. Jungnickel and C. Wetterich, Phys. Rev. D53, 5142 (1996)

${ }^{15}$ Y. Nambu and G. Jona-Lasinio, Phys. Rev. 122, 345 (1961)

${ }^{16}$ T. H. R. Skyrme, Proc. Roy. Soc. London Ser. A260, 127 (1961); Nucl. Phys. 31, 556 (1962)

17 A. Chodos, R. L. Jaffe, K. Johnson, C. .B. Thorn and V. F. Weisskopf, Phys. Rev. D12, 2060 (1975) 
A.7 Negative-Parity Baryon Resonances from Lattice QCD, F.X. Lee, D.B. Leinweber (U. of Adelaide).

It is well recognized that the investigation of processes involving the production of excited states of the nucleon $\left(\mathrm{N}^{*}\right)$ can provide relevant information on the non-perturbative regime of QCD. In the next years, the issue of $\mathrm{N}^{*}$ physics will be addressed by planned experiments at the Jefferson Lab, which will provide new experimental data in the so-called resonance region with an unprecedented accuracy. There is increasing demand for theoretical understanding of the $N^{*}$ properties from first principles calculations, such as lattice QCD. To our knowledge, there has been no lattice studies of odd-parity $N^{*}$ states to date.

In this work, we carry out exploratory calculations for odd-parity spin-1/2 $\left(J^{P}=1 / 2^{-}\right)$ and odd-parity spin-3/2 $\left(J^{P}=3 / 2^{-}\right)$baryon resonances on the lattice using improved actions. A lattice calculation of the masses of these baryon resonances will give us the first insights into the structure of orbitally excited baryons as predicted by QCD. In particular, the electromagnetic properties of these resonances may be completely different from model expectations if gluons carry a significant fraction of the angular momentum usually attributed to quark degrees of freedom alone. On the lattice, the excitation energies of the $1 / 2^{-}$baryons can be extracted from the two-point correlation function in the $Q C D$ vacuum

$$
G(\mathbf{p}, t)=\sum_{\mathbf{x}} e^{-i \mathbf{p} \cdot \mathbf{x}}\left\langle 0\left|\bar{\chi}_{\mathbf{1} / 2}^{-}(x) \bar{\chi}_{1 / 2}^{-}(0)\right| 0\right\rangle
$$

where $\chi_{1 / 2}^{-}$is the interpolating field used to excite the hadron in question. For the $1 / 2^{-}$ nucleon resonance, we use the following two interpolating fields

$$
\begin{gathered}
\chi_{1}^{-}(x)=\epsilon^{a b c}\left(u^{T a}(x) C \gamma_{5} d^{b}(x)\right) \gamma_{5} u^{c}(x) . \\
\chi_{2}^{-}(x)=\epsilon^{a b c}\left(u^{T a}(x) C d^{b}(x)\right) u^{c}(x) .
\end{gathered}
$$

The interpolating fields for other members of the $1 / 2^{-}$octet can be written down in a similar fashion. It is worth pointing out that these interpolating fields, despite having explicit negative-parity, can couple to both the positive and the negative states. Therefore the odd-parity states of the spin- $1 / 2$ baryon octet are not the lowest lying states. The parity of the state must be projected. This is done in the following manner.

The ability of the interpolating fields to couple to the states can be described by a phenomenological parameter $\lambda$, defined by

$$
\begin{aligned}
& \left\langle 0\left|\chi_{1 / 2}^{-}(0)\right| N^{+}\right\rangle=\lambda_{+} \gamma_{5} u(p), \\
& \left\langle 0\left|\chi_{1 / 2}^{-}(0)\right| N^{-}\right\rangle=\lambda_{-} u(p),
\end{aligned}
$$

where $u(p)$ denotes a spinor. By inserting a complete set of intermediate states, the correlation function can be written in the following form in the large Euclidean time limit

$$
\begin{aligned}
G(\mathbf{p}, t \rightarrow \infty) & =\sum_{s}\left\langle 0\left|\chi_{1 / 2}^{-}(x)\right| N_{s}^{ \pm}\right\rangle\left\langle N_{s}^{ \pm}\left|\bar{\chi}_{1 / 2}^{-}(0)\right| 0\right\rangle e^{-E_{ \pm}(\mathbf{p}) t} \\
& =\lambda_{+}^{2} \frac{\gamma \cdot p-M_{+}}{2 M_{+}} e^{-E_{+}(\mathbf{p}) t}+\lambda_{-}^{2} \frac{\gamma \cdot p+M_{-}}{2 M_{-}} e^{-E_{-}(\mathbf{p}) t}
\end{aligned}
$$


where $p$ is on shell and $E_{ \pm}(\mathrm{p})$ is the on-shell energy $\left(\mathrm{p}^{2}+M_{ \pm}^{2}\right)^{1 / 2}$. The large Euclidean time evolution of the two-point function will ultimately be dominated by the lower-lying even-parity state (the first term). At zero momentum, the odd-parity state can be projected out by taking the trace of $G(\mathbf{p}, t)$ with

$$
\Gamma_{4} \equiv \frac{1}{4}\left(1+\gamma_{4}\right)=\frac{1}{2}\left(\begin{array}{ll}
\text { I } & 0 \\
0 & 0
\end{array}\right) .
$$

Hence only the first two diagonal elements of the $4 \times 4$ matrix $G(\mathrm{p}, t)$ are needed to extract the mass of the odd-parity state. At finite momentum, the odd-parity excitation energy can be projected out by taking trace with

$$
\Gamma_{4}(\vec{p}) \equiv 1 / 4\left(1+\left(m_{+} / E_{+}\right) \gamma_{4}\right) .
$$

Note that this matrix is still diagonal so that only the four diagonal elements of $G(\mathbf{p}, t)$ are needed to project out odd-parity at finite momenta. This is only a factor of two in computation compared to the zero momentum case.

Mass ratios of the $1 / 2^{-}$states to the nucleon were calculated at several quark masses and extrapolated to the chiral limit. Table I shows the preliminary results based on 100 configurations. Clear separations of the odd-parity states from the ground state nucleon are displayed. Furthermore, it appears $\chi_{1}^{-}$couples mostly to the ground state in the $1 / 2^{-}$ spectrum, while $\chi_{2}^{-}$mostly to the next state. This is further supported by the fact that the overlap between $\chi_{1}^{-}$and $\chi_{2}^{-}$are smaller by more than a factor of 4 compared to the diagonal

\begin{tabular}{|c|c|c|c|c|c|}
\hline Ratio & $\frac{\pi}{\rho}=0.82$ & $\frac{\pi}{\rho}=0.78$ & $\frac{\pi}{\rho}=0.73$ & Chiral & Expt \\
\hline$\frac{N}{N(1535)}$ & $0.89(4)$ & $0.85(5)$ & $0.84(6)$ & $0.71(10)$ & 0.61 \\
\hline$\frac{N}{N(1650)}$ & $0.77(5)$ & $0.71(6)$ & $0.62(9)$ & $0.42(11)$ & 0.57 \\
\hline $\begin{array}{l}\text { Octet } \\
\Lambda(1650)\end{array}$ & $0.89(5)$ & $0.85(5)$ & $0.81(6)$ & $0.65(8)$ & 0.56 \\
\hline$\frac{N}{\Lambda(1800)}$ & $0.77(5)$ & $0.71(6)$ & $0.64(9)$ & $0.45(9)$ & 0.52 \\
\hline$\frac{N}{\Lambda(1405)}$ & $0.87(5)$ & $0.83(6)$ & $0.80(7)$ & $0.66(10)$ & 0.67 \\
\hline$\frac{N}{\Lambda(1650)}$ & $0.80(5)$ & $0.76(6)$ & $0.71(7)$ & $0.54(9)$ & 0.56 \\
\hline$\frac{N}{\Sigma(1620)}$ & $0.91(5)$ & $0.85(5)$ & $0.81(6)$ & $0.62(7)$ & 0.58 \\
\hline$\frac{N}{\Sigma(1750)}$ & $0.76(6)$ & $0.71(6)$ & $0.67(8)$ & $0.51(9)$ & 0.54 \\
\hline$\frac{N}{\Xi(1620)}$ & $0.90(5)$ & $0.85(5)$ & $0.81(6)$ & $0.63(5)$ & 0.58 \\
\hline $\begin{array}{l}=\frac{N}{1020)} \\
\equiv(1690)\end{array}$ & $0.77(6)$ & $0.71(6)$ & $0.66(7)$ & $0.48(8)$ & 0.56 \\
\hline
\end{tabular}
correlations.

TABLE I. Mass ratios of the $1 / 2^{-}$baryons to the nucleon extracted on the lattice based on 100 configurations. The errors are statistical only. The three pion to rho mass ratios correspond to quark masses of approximately $210,180,90 \mathrm{MeV}$. These ratios are extrapolated to the chiral limit linearly in the quark mass and compared with those in nature. The assignment of the states is our conjecture. 
The interpolating field for a $3 / 2^{-}$nucleon is unique

$$
\chi_{\mu}^{-}(x)=\epsilon^{a b c}\left(u^{T a}(x) C \gamma_{5} \gamma^{\tau} d^{b}(x)\right)\left[g_{\mu \tau}-\frac{1}{4} \gamma_{\mu} \gamma_{\tau}\right] \gamma_{5} u^{c}(x) .
$$

This interpolating field couples to both spin- $1 / 2$ and spin- $3 / 2$ baryons. Projection of the spin- $3 / 2$ component is required to remove ground state contaminations and to remove the nearby $1 / 2^{-}$nucleon state. The computational demand increases dramatically relative to the proton, almost a factor of 800 . It is currently in production on the C90 at NERSC. We have collected 25 configurations so far. Preliminary analysis indicates a splitting from the ground state nucleon, an encouraging result.

One obvious way to improve the signal is to increase statistics, perhaps to 500 to 1000 configurations in order to achieve the same level of statistical uncertainty as the nucleon. Another line of research is to use anisotropic actions with larger lattice spacing in the spatial dimensions than in the time dimension. It has been demonstrated in glueball mass calculations [4] that anisotropic actions with tadpole improvement can greatly improve the extraction of higher mass states. Nevertheless, this exploratory study showed promise in extracting $N^{*}$ structure from lattice QCD. After the spectrum of the odd-parity states are understood, we plan to move onto quantities of more phenomenological interest, such as the $N \rightarrow N^{*}$ electromagnetic transition form factors.

[1] F.X. Lee, D.B. Leinweber, hep-lat/9711044.

[2] Chung, Dosch, Kremer, Schall, Nucl. Phys. B197, 55 (1982).

[3] D.B. Leinweber, Phys. Rev. D51, 6383 (1995).

[4] C. Morningstar and M. Peardon, hep-lat/9704011. 


\section{A.8 The $N \rightarrow \Delta$ Electromagnetic Transition Amplitudes from QCD Sum} Rules, F.X. Lee.

Electromagnetic transition amplitudes encode sensitive dynamical information on the structure of hadrons. Experimental determination of these amplitudes have become more and more accurate in recent years and will become even better with experiments planned at the Jefferson Lab. There is increasing demand for theoretical understanding of the transitions based on first principles. In this work, we present a systematic calculation of the transition amplitudes in the QCD sum rule approach [1]. Such a calculation would help establish the connection with the non-perturbative structure of the QCD vacuum, and to our knowledge has not been attempted within the approach. The goal is two-fold. First, we want to find out if the approach can be successfully applied to these observables by carrying out an explicit calculation. Second, we want to achieve some realistic understanding of the uncertainties involved in such a determination by employing a Monte-Carlo based analysis procedure. This would help us assess the limitations and find ways for improvements.

The object of study is the two-point correlation function in the QCD vacuum in the presence of a constant background electromagnetic field $F_{\mu \nu}$ :

$$
\Pi_{\alpha}(p)=i \int d^{4} x e^{i p \cdot x}\left\langle 0\left|T\left\{\eta_{\alpha}^{\Delta}(x) \bar{\eta}^{N}(0)\right\}\right| 0\right\rangle_{F},
$$

where $\eta^{N}$ and $\eta_{\alpha}^{\Delta}$ are the interpolating fields (or currents) for the octet and decuplet baryon under consideration, respectively. Note that we will use $N$ and $\Delta$ in a generic sense to represent all possible transitions from the octet to the decuplet. The QCD sum rules are derived by calculating the linear response of the correlator using Operator-Product-Expansion (OPE), on the one hand, and matching it to a phenomenological representation, on the other. The calculation is similar in spirit to the one for decuplet baryon magnetic moments in Ref. [2] in which a lot of the details needed here can be found. Here we only want to give the phenomenological representation of the transitions.

The electromagnetic $N \rightarrow \Delta$ transition vertex is defined by the current matrix element [3]

$$
\left\langle\Delta k^{\prime} s^{\prime}\left|J^{\mu}(0)\right| N k s\right\rangle=i \sqrt{\frac{2}{3}} \bar{u}_{\tau}\left(k^{\prime}, s^{\prime}\right) \Gamma^{\tau \mu}(q, P) u(k, s),
$$

where $q=k^{\prime}-k$ and $P=\left(k^{\prime}+k\right) / 2$. Note that we are only concerned with real photons $\left(q^{2}=0\right)$. The tensor $\Gamma^{\tau \mu}$ satisfies the requirements of Lorentz and gauge invariance, and can be parameterized by two constants. Historically, there exist two sets of definitions: $G_{1}$, $G_{2}$, and $G_{E}$ and $G_{M}$, which are analogs of the Dirac and Sachs form factors for the $\gamma N N$ vertex, respectively. Both sets are in units of particle's natural magnetons. The two sets are related by

$$
\begin{aligned}
& G_{1}=\frac{3 M_{\Delta}}{2 M_{N}\left(M_{\Delta}+M_{N}\right)}\left(G_{M 1}-G_{E 2}\right), \\
& G_{2}=\frac{3}{2 M_{N}\left(M_{\Delta}+M_{N}\right)}\left(-G_{M 1}+\frac{3 M_{\Delta}+M_{N}}{M_{\Delta}-M_{N}} G_{E 2}\right) .
\end{aligned}
$$

Note that $G_{2}$ is much more sensitive to $G_{E 2}$ than $G_{1}$ since the $G_{E 2}$ in Eq. (13) is magnified by a large factor that depends on the difference of the masses. There exist other definitions. In the conventions of the Particle Data Group, 


$$
\begin{aligned}
f_{M 1} & =\frac{e}{2 M_{N}}\left(\frac{M_{\Delta}^{2}-M_{N}^{2}}{2 M_{N}}\right)^{1 / 2} G_{M 1}, \\
f_{E 2} & =-\frac{e}{2 M_{N}}\left(\frac{M_{\Delta}^{2}-M_{N}^{2}}{2 M_{N}}\right)^{1 / 2} G_{E 2} .
\end{aligned}
$$

The ratio of the electric quadrupole and the magnetic dipole transition moments is defined by

$$
R_{E M} \equiv \frac{f_{E 2}}{f_{M 1}}=-\frac{G_{E 2}}{G_{M 1}} .
$$

The helicity amplitudes are defined by

$$
\begin{aligned}
& A_{1 / 2}=-\left(f_{M 1}+3 f_{E 2}\right) / 2, \\
& A_{3 / 2}=-\sqrt{3}\left(f_{M 1}-f_{E 2}\right) / 2 .
\end{aligned}
$$

The current values of $A_{1 / 2}=-141 \pm 5 A_{3 / 2}=-257 \pm 8$ for $\gamma p \rightarrow \Delta^{+}$translate into $f_{M 1} \approx 293, f_{E 2} \approx-37$ (both in units of $10^{-3}(G e V)^{-1 / 2}$ ), and $G_{M}=3.12, G_{E}=0.04$, and $G_{1}=2.8(\mathrm{GeV})^{-1}, G_{2}=-1.6(\mathrm{GeV})^{-2}$. In this work, it is most convenient to compute $G_{1}$ and $G_{2}$ because they are comparable in magnitude and opposite in sign. Then it is a easy matter to convert to other conventions.

We have successfully derived a complete set of QCD sum rules for the transition amplitudes. Here we present some relations we found that exist among the transition moments in different channels that directly result from the underlying symmetries. The following relations are exact:

$$
\begin{aligned}
\gamma p \rightarrow \Delta^{+} & =-\gamma n \rightarrow \Delta^{0} . \\
\Sigma^{0} & =\frac{\Sigma^{+}+\Sigma^{-}}{2} .
\end{aligned}
$$

The following relations reflect subtle symmetry breakings in the strange quark:

$$
\begin{aligned}
& \gamma \Sigma^{-} \rightarrow \Sigma^{*-} \approx-\gamma \Xi^{-} \rightarrow \Xi^{*-}, \\
& \gamma \Sigma^{+} \rightarrow \Sigma^{*+} \approx-\gamma \Xi^{0} \rightarrow \Xi^{* 0} .
\end{aligned}
$$

The following moments vanish under $\mathrm{SU}(3)$ flavor symmetry:

$$
\begin{array}{r}
\gamma \Sigma^{-} \rightarrow \Sigma^{*-}=0, \\
\gamma \Xi^{0} \rightarrow \Xi^{* 0}=0 .
\end{array}
$$

Numerical analysis using the Monte-Carlo procedure is under progress.

[1] M.A. Shifman, A.I. Vainshtein and Z.I. Zakharov, Nucl. Phys. B147, 385, 448 (1979).

[2] F.X. Lee, Phys. Rev. D 57, 1801 (1998).

[3] D.B. Leinweber, T. Draper, and R.M. Woloshyn, Phys. Rev. D46, 3067 (1992). 
A.9 Higher Nucleon Resonances in Exclusive Reactions $(\gamma, \pi N)$ on Nuclei, F.X. Lee, C. Bennhold (George Washington U.), S.S. Kamalov (Mainz) and L.E. Wright (Ohio U.).

Meson photoproduction has been a valuable source of information on the structure of hadrons and nuclei. With the advent of the new generation of high-intensity continuouswave electron accelerators, as represented by the Jefferson Lab, the field reaches a new level of promise. The new facilities allow exclusive measurements with unprecedented precision under a variety of kinematical conditions, thus making it possible to study higher excited states of hadrons in greater detail.

Quasifree pion photoproduction allows for the study of the production process in the nuclear medium as well as final state interaction effects without being obscured by the details of the nuclear transition. This is due mainly to the quasifree nature of the reaction which permits the kinematic flexibility to have small momentum transfers. Conceptually, the initial nucleus is a target holder which presents a bound nucleon to the incoming photon beam. The basic reaction $N(\gamma, \pi N)$ takes place in the nuclear medium producing a continuum pion and nucleon which interact with the residual nucleus as they exit the target. The key ingredients in such a description are: a) the single-particle wave function of the initial nucleon and spectroscopic factor, usually taken from electron scattering, b) the elementary pion photoproduction amplitudes, obtained from measuring the free processes, c) the nucleonnucleus final state interaction, taken from elastic scattering, and finally, d) the pion-nucleus final state interaction, also taken from elastic scattering.

Using a newly developed operator [1], we carry out nuclear calculations in this work for the exclusive reactions, $A(\gamma, \pi N) A-1$, beyond the first resonance region in a distorted wave impulse approximation (DWIA) framework. Pion photoproduction on a nucleus provides a different environment in which the reaction can take place. Issues arise such as the nuclear reaction mechanism, the interaction of the resonances with the medium, and the final state interactions. Our focus is on the puzzle of the 'damped resonances' in the second and third resonance regions as seen in inclusive photoabsorption cross section data on various nuclei [2]. The data show an unexpected damping behavior of the higher resonances when compared with the same process on the proton and the deuteron. Clearly, in order to isolate the mechanism for this mysterious phenomenon the individual exclusive channels need to be investigated.

The elementary operator contains contributions from the resonances $P_{33}(1232)$, $P_{11}(1440), D_{13}(1520), D_{33}(1740), S_{11}(1535), S_{11}(1650)$, and $F_{15}(1680)$, in addition to the usual Born plus vector meson contributions. It gives a good description of single pion photoproduction data up to about $1.1 \mathrm{GeV}$. Final state interactions are incorporated via optical potentials and are found to be substantial in the coincidence cross sections, but insensitive to the photon asymmetry.

The insensitivity of the photon asymmetry to FSI and to nuclear structure opens the way to use this observable to study possible modifications of the basic production process in the nuclear medium. Such modifications may manifest themselves through variations in the parameters of the resonance such as the mass, the width and the coupling constant (strength). It is expected that its mass would be decreased from interactions via a nuclear potential. Additional channels might open in the medium, causing an increase in its decay width. The coupling at the interaction vertices might also be modified. To perform a 
phenomenological sensitivity study of the $D_{13}$ and $F_{15}$ resonances, we apply a $3 \%$ decrease in their masses and a $60 \%$ broadening of their widths in the elementary operator. These values are in line with those suggested by an cooperative damping model of the resonances [3]. As for the strength, we use the values suggested by a quark exchange model [4]: a reduction of the $D_{13}$ strength by $12 \%$ and the $F_{15}$ strength by $22 \%$. We do not claim any validity of these models, but are mostly interested in exploring sensitivity of the observables to possible changes in the resonance parameters. Rigorous studies of such effects clearly require a more dynamical treatment, as in the delta-hole model.

Fig. 1 shows the effects of medium modifications for the process ${ }^{12} C\left(\gamma, \pi^{-} p\right)^{11} C_{\text {g.s. }}$. The four curves correspond to the full results (solid), the results with the mass decreased by $3 \%$ (dotted), with the width increased by $60 \%$ (dashed), and with the strength decreased by $12 \%$ for $D_{13}$ and $22 \%$ for $F_{15}$ (dot-dashed). The results are most sensitive to changes in the resonance mass, followed by the resonance width. The sensitivity to the resonance strength is surprisingly small. In the cross sections, the small reduction of the $D_{13}$ mass results in a large shift of the second peak as well some reduction in the energy distributions, and a large reduction in the angular distributions. The effects of the change in width are also quite large. As for the 'cleaner' photon asymmetry, there is a noticeable effect due to the mass change for the $D_{13}$ in the energy distribution, and a very large sensitivity to the $F_{15}$ in the angular distribution. These effects are large enough to be detected by experiments. Especially if the photon asymmetry is measured, the effects can easily be detected by experiments. Indeed, extensive measurements of the reaction in all four channels have been planned for Hall B at TJNAF [5], covering a wide range of kinematics. We therefore believe that measurements of exclusive quasifree pion photoproduction with polarized photons are an important future step that should help untangle the riddle of the damped resonances.

[1] D. Drechsel, O. Hanstein, S.S. Kamalov, and L. Tiator, Mainz preprint MKPH-T-98-10, nucl-th/9807001.

[2] N. Bianchi et. al., Phys. Lett. B229, 219 (1993), Phys. Lett. B309, 5 (1993), Phys. Lett. B325, 333 (1993), Phys. Rev. C 54, 1688 (1996); M. Anghinolfi et. al., Phys. Rev. C 47, R992 (1993). M. MacCormick et. al., Phys. Rev. C 55, 1037 (1997).

[3] M. Hirata, K. Ochi, and T. Takaki, nucl-th/9711017.

[4] M.M. Giannini and E. Santopinto, Phys. Rev. C 49, R1258 (1994).

[5] CEBAF E89-027, L.B. Weinstein, private communication. 

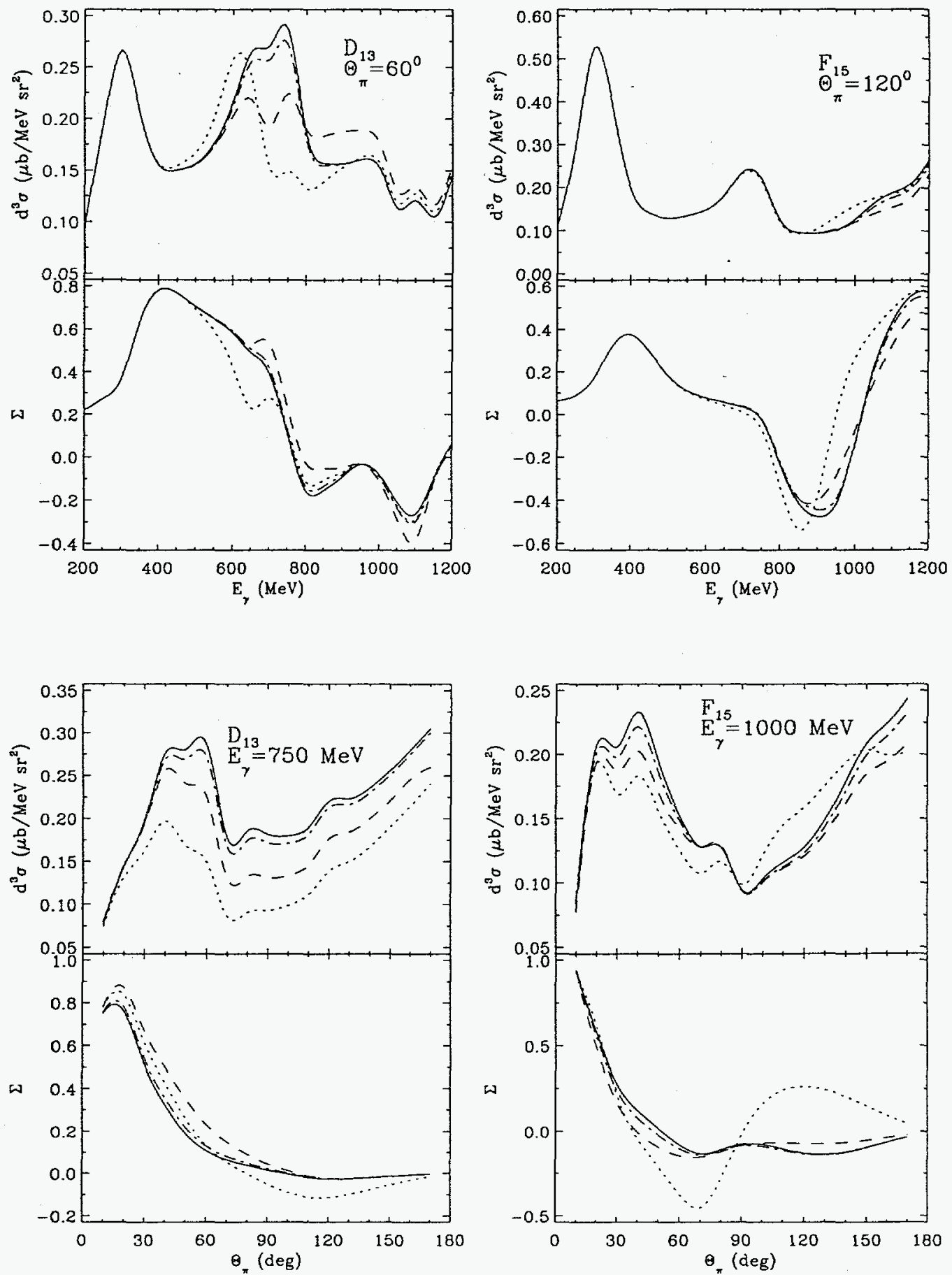

FIG. 1. Effects of possible medium modification of the $D_{13}(1520)$ and $F_{15}(1680)$ resonances in the reaction ${ }^{12} C\left(\gamma, \pi^{-} p\right)^{11} C_{g . s .}$. The four curves correspond to the full results (solid), with the mass decreased by $3 \%$ (dotted), with the width increased by $60 \%$ (dashed), and with the strength decreased by $12 \%$ for $D_{13}$ and $22 \%$ for $F_{15}$ (dot-dashed). The calculations were done in DWIA. 
B PUBLICATIONS AND REPORTS-October 1, 1997 to August 1, 1998

1. Published Articles

(a) Electroweak Excitation of the Delta: Hadron Structure and New Physics N.C. Mukhopadhyay, M.J. Ramsey-Musolf, S.J Pollock, J. Liu, H.-W. Hammer, Nuclear Physics A633 (1998) 481; nucl-th/9801025.

(b) Monte Carlo and Renormalization Group Effective Potentials in Scalar Field Theories at Finite Temperature J.D.Shafer and J.R.Shepard, Phys. Rev. D55, 4990-4996 (1997).

(c) A Plaquette Basis for the Study of Heisenberg Ladders, J.Piekarewicz and J.R.Shepard, Phys. Rev. B56, 5366-5372 (1997); cond-mat/9612139.

(d) Dynamic Spin Response for Heisenberg Ladders, J.Piekarewicz and J.R.Shepard, Phys. Rev. B57, 10260-10263 (1998); cond-mat/9707207.

(e) Renormalization Group Flow Equations for the Sigma Model, A.S.Johnson, J.A.McNeil and J.R.Shepard, Phys. Rev. D58:014001 (1998); hep-ph/9711370.

(f) Predictive Ability of QCD Sum rules for Decuplet Baryons, F.X. Lee, Phys. Rev. C57, 322 (1997).

(g) Magnetic Moments of $\Delta^{++}$and $\Omega^{-}$from QCD Sum Rules, F.X. Lee, Physics Letters B419, 14 (1998).

(h) Determination of Decuplet Baryon Magnetic Moments from QCD Sum Rules, F.X. Lee, Phys. Rev. D57, 1801 (1998).

\section{Articles Accepted or Submitted for Publication}

(a) Parity-Violating $\Delta(1232)$ Electroweak Production: Axial Structure and New Physics, S. J. Pollock et al., subm. to Few-Body Systems Suppl.; nucl-th/9806079

(b) Perturbation Theory for Spin Ladders Using Angular-Momentum Coupled Bases, J.Piekarewicz and J.R.Shepard, Phys. Rev. B, in press; cond-mat/9804261.

(c) Application of Racah Algebra to Quantum Spin Systems, J.Piekarewicz and J.R.Shepard, to appear in Revista Mexicana de Fisica.

(d) Finite Temperature Renormalization Group Effective Potentials for the Linear Sigma Model, J.D. Shafer and J.R. Shepard, subm. to Phys. Rev. D.

(e) Light Hadron Spectroscopy on Coarse Lattices with $O\left(a^{2}\right)$ Mean-Field Improved Actions, F.X. Lee and D.B. Leinweber, to appear in Phys. Rev. D.

(f) Higher Nucleon Resonances in Exclusive Reactions $(\gamma, \pi N)$ on Nuclei, F.X. Lee, C. Bennhold, S.S. Kamalov and L.E. Wright, subm. to Phys. Rev. C.

(g) A High Resolution ${ }^{16} O\left(\gamma, \pi^{-} p\right)$ Experiment, M.A. van Uden, E.C. Aschenauer, L.J. de Bever, H.L. Castricum, S. Frullani, E. Cisbani, F. Garibaldi, 
D.L. Groep, M. Iodice, W-J.Kasdorp, F.X. Lee, C.J.G. Onderwater, M. Schroevers, C.M. Spaltro, R. Starink, G. van der Steenhoven, J.J.M. Steijger, G.M. Urciuoli, and L.E. Wright, subm. to Phys. Rev. C.

3. Invited and Contributed Papers and Abstracts

(a) Theoretical issues in neutral weak interactions involving atoms and nuclei, S.J. Pollock, Invited talk, abstract published in Bulletin of the APS, Vol 42, No. 7 (DNP97 Oct 5-8, Whistler B.C.)

(b) Theoretical considerations in Parity Violating $\left(\vec{e}, e^{\prime}\right)$ Nucleus Scattering, M. Welliver and S. J. Pollock, Abstract and talk for Spring '98 American Physical Society meeting, 4-Corners Section.

(c) Parity-Violation $\Delta$ Electroweak Production: Hadron Structure and

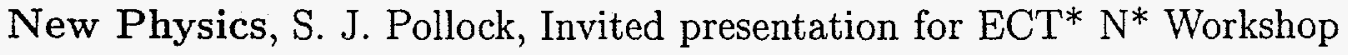

(d) New Results for Heisenberg Spin Ladders Using a Plaquette Basis, J.R. Shepard, Abstract and talk published in Bulletin of the APS, Vol 42, No. 7 (DNP97 Oct 5-8, Whistler B.C.), p. 1678.

(e) A Nuclear Physics Approach to High-Temperature Superconductivity, J. Piekarewicz and J.R. Shepard, Invited Talk at a Special Theoretical Symposium in Honor of D. Robson on the Occasion of His 60th Birthday, Athens, GA, September 11-12, 1997; presented by J. Piekarewicz.

(f) Improved Lattice QCD Actions for Hadron Phenomenology, D.B. Leinweber and F.X. Lee, to appear in Proceedings of the Workshop on Methods in Non-Perturbative Field Theory, Adelaide, February 2-13, 1998.

(g) Quasifree Kaon Photoproduction as a Probe of Hyperon-Nucleus Interactions, F.X. Lee, L.E. Wright and C. Bennhold, Contributed talk at the APS Spring Meeting, Columbus, Ohio, April 18-21, 1998.

(h) Negative-Parity Baryon Resonances from Lattice QCD, F.X. Lee and D.B. Leinweber, Contributed talk at Lattice'98, Boulder, CO, July 13-18, 1998.

(i) Quasifree $\pi$ and $\eta$ Photoproduction on Nuclei, F.X. Lee, Invited talk at Gordon Conference on Photonuclear Reactions, Tilton NH, Jul. 26-31, '98.

\section{PERSONNEL}

1. Academic and Scientific

P.D. Kunz

J.R. Shepard

S.J. Pollock

F.X. Lee
Emeritus Professor

Professor

Assistant Professor

Postdoctoral Researcher

2. Research Assistants

J. Shafer

M. Welliver 